\title{
On Notions of Detectability and Observers for Hybrid Systems
}

\author{
Pauline Bernard ${ }^{\mathrm{a}}$, Ricardo G. Sanfelice ${ }^{\mathrm{b}}$ \\ ${ }^{a}$ Centre Automatique et Systèmes, MINES Paris Tech, Université PSL, France. \\ ${ }^{b}$ Department of Electrical and Computer Engineering, University of California, Santa Cruz, CA 95064, USA.
}

\begin{abstract}
Notions of observer and detectability are well established for continuous-time and discrete-time systems, and are known to be linked, since a system must be detectable to admit an observer. Unfortunately, defining such notions for a hybrid system is not straightforward because solutions do not share the same hybrid time domain. In this paper, we propose to define observers and detectability for hybrid systems, such that detectability is still necessary for the existence of an observer and such that standard definitions are recovered for continuous-time and discrete-time systems, when seen as special cases of hybrid systems. We rely on a recent definition of hybrid systems with hybrid inputs and use jump reparametrizations to define convergence and equality of outputs.
\end{abstract}

Keywords: observer, hybrid systems, detectability

\section{Introduction}

\subsection{Context}

For continuous-time (CT) and discrete-time (DT) systems, asymptotic observers are commonly defined as dynamical systems taking the plant's output as input and whose state asymptotically converges to the plant's state. The existence of such an object then requires some intrinsic properties of the plant, in particular that the plant be asymptotically detectable: the trajectories giving the same output must asymptotically converge to one another [1]. However, those notions are not straightforward to define for a hybrid plant, since they involve comparisons of hybrid solutions defined on different hybrid time domains.

When the jump times of the plant are assumed to be known, the difficulties due to a possible mismatch of the trajectories' domains disappear since the observer can be synchronized with the plant. Similarly, notions of detectability, observability and determinability reduce to comparing outputs with the same time domain $([2,3,4])$. However, when the plant's jump times are unknown, the observer must be a hybrid system which does not necessarily jump at the same time as the plant. This difficulty is avoided in [5] thanks to a change of coordinates transforming the jump map into the identity map and thus somehow making the jumps disappear in the observer. As for [6], an extended system containing both the plant and the observer is directly analyzed. In the particular setting of switched systems, the problem is handled by estimating the switching signal $([7,8,9,10])$, whose observability has been studied in $[11,12]$.

Apart from definitions of zero-detectability [13, 14], we are not aware of general notions of (incremental) detectability and observers in the literature for general hybrid systems. This paper thus proposes to define such notions, building from recent definitions of hybrid systems with hybrid inputs $[15,16]$ and the literature of hybrid reference tracking [17] and hybrid incremental stability [18, 19], where methods for the comparison of hybrid solutions were also introduced.

Email addresses: pauline.bernard@mines-paristech.fr (Pauline Bernard), ricardo@ucsc.edu (Ricardo G. Sanfelice) 


\subsection{Detectability and Observers for CT Systems}

Consider a CT system

$$
\dot{x}=f(x) \quad, \quad y=h(x)
$$

initialized in a set of interest $\mathcal{X}_{0}$. We usually define an observer as a dynamical system of the form

$$
\dot{z}=F(z, y) \quad, \quad \hat{x}=H(z, y)
$$

initialized in a set $\mathcal{Z}_{0}$ and whose complete solutions, i.e. solutions defined on $\mathbb{R}_{\geq 0}$, are asked to verify stability and convergence properties. If $z$ lives in the same space as $x$, we may directly take $\hat{x}=z$, but that is not necessarily the case for nonlinear systems. The existence of such an observer then intrinsically requires some detectability properties of the system (1): the output $y$ should somehow contain enough information to uniquely determine the plant's state.

Definition 1.1. The system (2) is an asymptotic observer of (1) on $\mathcal{X}_{0}$ if there exists a (known) set of initial conditions $\mathcal{Z}_{0}$ such that for any complete solution $x$ of $(1)$ initialized in $\mathcal{X}_{0}$, any maximal solution $z$ of (2) initialized in $\mathcal{Z}_{0}$ with input $y=h(x)$ is also complete and verifies $\lim _{t \rightarrow+\infty}|x(t)-\hat{x}(t)|=0$.

Definition 1.2. The system (1) is asymptotically detectable on $\mathcal{X}_{0}$ if any pair of complete solutions $x_{a}$ and $x_{b}$ of (1) initialized in $\mathcal{X}_{0}$ such that

$$
h\left(x_{a}(t)\right)=h\left(x_{b}(t)\right) \quad \forall t \in \mathbb{R}_{\geq 0}
$$

verifies $\lim _{t \rightarrow+\infty}\left|x_{a}(t)-x_{b}(t)\right|=0$.

In other words, trajectories that have the same outputs converge to each other. As shown for instance in [1], this detectability property is necessary for the existence of an observer.

Theorem 1.3. If system (1) admits an asymptotic observer on $\mathcal{X}_{0}$, then system (1) is asymptotically detectable on $\mathcal{X}_{0}$.

Proof Consider complete solutions $x_{a}$ and $x_{b}$ of $(1)$ such that $h\left(x_{a}(t)\right)=h\left(x_{b}(t)\right)$ for all $t \geq 0$. Take a maximal solution $z$ of (2) initialized in $\mathcal{Z}_{0}$ with input $y=h\left(x_{a}\right)$. Then, by definition, $z$ is complete and $\lim _{t \rightarrow+\infty}\left|x_{a}(t)-\hat{x}(t)\right|=0$ with $\hat{x}=H\left(z, h\left(x_{a}\right)\right)$. But since $h\left(x_{a}\right)=h\left(x_{b}\right), z$ is also solution of (2) with input $y=h\left(x_{b}\right)$, and thus $\lim _{t \rightarrow+\infty}\left|x_{b}(t)-\hat{x}(t)\right|=0$ with $\hat{x}=H\left(z, h\left(x_{a}\right)\right)=H\left(z, h\left(x_{b}\right)\right)$. It thus follows by triangle inequality that $\lim _{t \rightarrow+\infty}\left|x_{a}(t)-x_{b}(t)\right|=0$.

A similar result can be obtained in the same way for DT systems.

Remark 1.4. Definitions 1.1 and 1.2 require only asymptotic convergence of $\hat{x}-x$ or $x_{a}-x_{b}$ to zero for complete solutions. In other words, they only deal with attractivity. However, we may sometimes want to require stronger properties of the observer (stability, finite-time convergence, tunable speed of convergence, etc.) and whichever constraint we add in the observer, then imposes stronger properties of the system that could be translated in a stronger notion of detectability with the same reasoning as Theorem 1.3.

\subsection{Towards Hybrid Systems}

Consider now a general hybrid system

$$
\mathcal{H}\left\{\begin{array}{rl}
\dot{x} \in f(x) & x \in C \\
x^{+} \in g(x) & x \in D
\end{array} \quad, \quad y=h(x)\right.
$$

with state $x \in \mathbb{R}^{d_{x}}$ and output $y \in \mathbb{R}^{d_{y}}$. The solutions are now hybrid $\operatorname{arcs}(t, j) \mapsto x(t, j)$ defined on a hybrid time domain $\operatorname{dom} x \subseteq \mathbb{R}_{\geq 0} \times \mathbb{N}$ according to [20], with both continuous-time evolution in $C$ and discrete events in $D$ (flows and jumps). 
If we want to properly define notions of observers and detectability as it has been done for CT/DT systems, we need to think about both definitions together in a way that ensures that detectability is a necessary condition for the existence of an observer. Besides, both CT and DT detectability/observers should be recovered as particular cases when $D=\emptyset$ and $C=\emptyset$ respectively. The main difficulties are as follows:

- The observer should be a hybrid system taking the (hybrid) output of $\mathcal{H}$ as input, but the latter has its own hybrid time domain that may differ from the hybrid time domain of the observer's solution. Hence, a more general notion of solution must be used;

- Trajectories do not share a common time domain. This makes the comparison between $x_{a}$ and $x_{b}$ for detectability, or between $x$ and $\hat{x}$ for observers, not straightforward;

- Completeness can happen either in the time-horizon (if $t$ goes to $+\infty$ ) or the jump horizon (if $j$ goes to $+\infty)$ and at different times;

- Asking for exact convergence of $\hat{x}$ to $x$ may be too restrictive around the jump times where an arbitrarily small mismatch of jump times between $\hat{x}$ and $x$ leads to a significant error if $g \neq \operatorname{Id}$, i.e., $g$ is not the identity map.

A notion of solutions to hybrid systems with hybrid inputs was proposed in [15, 16], relying on a socalled jump reparametrization. This process, recalled in Section 2, enables to compare $\hat{x}$ and $x$ on a common domain and thus to formulate a definition of asymptotic observers that resembles Definition 1.1. Note that more general notions of convergence will be allowed, with $(\hat{x}, x)$ required to converge to a set $\mathcal{A} \subset \mathbb{R}^{d_{x}} \times \mathbb{R}^{d_{x}}$ possibly larger than

$$
\mathcal{A}=\left\{(x, \hat{x}) \in \mathbb{R}^{d_{x}} \times \mathbb{R}^{d_{x}}: x=\hat{x}\right\} .
$$

Then, in order to determine whether two hybrid outputs are "equal" as in Definition 1.2, we propose in Section 3 an algorithm that enables to write two hybrid arcs on a common time domain and thus define asymptotic detectability relative to $\mathcal{A}$. Regarding the completeness condition, we will see that only complete solutions sharing the same time horizon need to be compared.

The link between these definitions and more intuitive extended systems is investigated in Section 4.

Finally, in Section 5, we show that the proposed definitions preserve the tight link between asymptotic detectability and observers exhibited for CT systems in Theorem 1.3, namely, we prove the following main result.

Theorem 1.5. Let $\mathcal{A}$ be a nonempty subset of $\mathbb{R}^{d_{x}} \times \mathbb{R}^{d_{x}}$. If $\mathcal{H}$ admits an asymptotic observer on $\mathcal{X}_{0}$ relative to $\mathcal{A}$, then $\mathcal{H}$ is asymptotically detectable on $\mathcal{X}_{0}$ relative to $\mathcal{A}$.

\subsection{Notations and Preliminaries}

We denote by $\mathbb{R}$ (resp. $\mathbb{N}$ ) the set of real (resp. natural) numbers, and $\mathbb{R}_{\geq 0}:=[0,+\infty), \mathbb{R}_{>0}:=(0,+\infty)$, and $\mathbb{N}_{>0}:=\mathbb{N} \backslash\{0\}$. For a set $\mathcal{S}, \operatorname{cl}(\mathcal{S})$ denotes its closure, int $(\mathcal{S})$ its interior, and card $\mathcal{S}$ its cardinality (possibly infinite).

The set of maximal solutions to a hybrid system $\mathcal{H}$ initialized in $\mathcal{X}_{0}$ is denoted $\mathcal{S}_{\mathcal{H}}\left(\mathcal{X}_{0}\right)$, or $\mathcal{S}_{\mathcal{H}}\left(\mathcal{X}_{0} ; u\right)$ if $\mathcal{H}$ takes $u$ as input. For a hybrid arc $(t, j) \mapsto \phi(t, j)$ defined on a hybrid time domain dom $\phi$, we denote $\operatorname{dom}_{t} \phi$ (resp. $\left.\operatorname{dom}_{j} \phi\right)$ its projection on the time (resp. jump) axis, and for a positive integer $j, t_{j}(\phi)$ the time stamp associated to the $j$ th jump (i.e., the only time satisfying $\left(t_{j}(\phi), j\right) \in \operatorname{dom} \phi$ and $\left.\left(t_{j}(\phi), j-1\right) \in \operatorname{dom} \phi\right)$, and $\mathcal{I}_{j}(\phi)$ the largest interval such that $\mathcal{I}_{j}(\phi) \times\{j\} \subseteq \operatorname{dom} \phi$. We define also $\mathcal{T}(\phi)=\left\{t_{j}(\phi): j \in \operatorname{dom}_{j} \phi \cap \mathbb{N}_{>0}\right\}$ as the set of jump times of $\phi, T(\phi)=\sup _{\operatorname{dom}} \phi \in \mathbb{R}_{\geq 0} \cup\{+\infty\}$ the maximal time of the domain, $J(\phi)=\sup _{j} \operatorname{dom}_{j} \phi \in \mathbb{N} \cup\{+\infty\}$ the total number of jumps, and, for a time $t$ in $\mathbb{R}_{>0}, \mathcal{J}_{t}(\phi)=\left\{j \in \mathbb{N}_{>0}: t_{j}(\phi)=t\right\}$ the set of jump counters associated to the jumps occurring at time $t$. It follows that card $\mathcal{J}_{t}(\phi)$ is the number of jumps of $\phi$ occurring at time $t$. A hybrid arc $\phi$ is said to be $t$-complete (resp. $j$-complete) if $\operatorname{dom}_{t} \phi$ (resp. $\left.\operatorname{dom}_{j} \phi\right)$ is unbounded, complete if dom $\phi$ is unbounded, and Zeno if it is complete with $\sup _{t} \operatorname{dom}_{t} \phi<\infty$. 
Finally, we will need to consider convergence to a subset $\mathcal{A}$ of $\mathbb{R}^{d_{x}} \times \mathbb{R}^{d_{x}}$. For that, a map $d_{\mathcal{A}}: \mathbb{R}^{d_{x}} \times \mathbb{R}^{d_{x}} \rightarrow$ $\mathbb{R}_{\geq 0}$ defines a distance relative to $\mathcal{A}$ if for all $x_{a}, x_{b}, x_{c}$ in $\mathbb{R}^{d_{x}}$,

$$
\begin{aligned}
& d_{\mathcal{A}}\left(x_{a}, x_{b}\right)=0 \quad \Longleftrightarrow \quad\left(x_{a}, x_{b}\right) \in \mathcal{A} \\
& d_{\mathcal{A}}\left(x_{a}, x_{b}\right)=d_{\mathcal{A}}\left(x_{b}, x_{a}\right) \\
& d_{\mathcal{A}}\left(x_{a}, x_{b}\right) \leq d_{\mathcal{A}}\left(x_{a}, x_{c}\right)+d_{\mathcal{A}}\left(x_{c}, x_{b}\right)
\end{aligned}
$$

\section{Hybrid Asymptotic Observers}

Inspired from (2), we define an observer as a hybrid system taking the plant's output $y$ as input and producing an estimate $\hat{x}$ of the plant's state as output, namely

$$
\hat{\mathcal{H}}\left\{\begin{array}{rlrl}
\dot{z} & \in F(z, y) & & (z, y) \in \hat{C} \\
z^{+} & \in G(z, y) & & (z, y) \in \hat{D}
\end{array} \quad, \quad \hat{x}=H(z, y)\right.
$$

with state $z \in \mathbb{R}^{d_{z}}$, such that " $\hat{x}$ converges to $x$ " in some sense. First, solutions to (7) must be defined with care because the hybrid input $y$ coming from the plant $\mathcal{H}$ has its own time domain and its jumps have no reason to happen when $(z, y)$ is in the jump set $\hat{D}$. Therefore, their jumps are not necessarily synchronized. Appropriate definitions have been given in [16] which we briefly recall next.

\subsection{Reparametrization and Definition of Solutions}

Definition 2.1 ([15, 16]). Given a hybrid arc $\phi$, a hybrid arc $\phi^{\mathrm{r}}$ is a $j$-reparametrization of $\phi$ if there exists a function $\rho: \mathbb{N} \rightarrow \mathbb{N}$ such that

$$
\begin{gathered}
\rho(0)=0 \quad, \quad \rho(j+1)-\rho(j) \in\{0,1\} \quad \forall j \in \mathbb{N} \\
\phi^{\mathrm{r}}(t, j)=\phi(t, \rho(j)) \quad \forall(t, j) \in \operatorname{dom} \phi^{\mathrm{r}} .
\end{gathered}
$$

The hybrid arc $\phi^{\mathrm{r}}$ is a full $j$-reparametrization of $\phi$ if

$$
\operatorname{dom} \phi=\bigcup_{(t, j) \in \operatorname{dom} \phi^{\mathrm{r}}}(t, \rho(j))
$$

The map $\rho$ is called $j$-reparametrization map from $\phi$ to $\phi^{\mathrm{r}}$.

In other words, $\phi^{\mathrm{r}}$ takes at each time $t$ the same values as $\phi$, but maybe associated to a different jump index: initially $\phi^{\mathrm{r}}(t, 0)=\phi(t, 0)$ for all $t \in \mathcal{I}_{0}\left(\phi^{r}\right)$, and when $\phi^{r}$ jumps,

- either $\rho(1)=1$ and $\phi^{\mathrm{r}}(t, 1)=\phi(t, 1)$ for all $t \in \mathcal{I}_{1}\left(\phi^{r}\right)$,

- or $\rho(1)=0$ and $\phi^{\mathrm{r}}(t, 1)=\phi(t, 0)$ for all $t \in \mathcal{I}_{1}\left(\phi^{r}\right)$,

and so on. This means that if $\rho(j+1)=\rho(j)+1$, the $j$ th jump of $\phi^{\mathrm{r}}$ corresponds to an actual jump in the domain of $\phi$, and if $\rho(j+1)=\rho(j), \phi^{\mathrm{r}}$ exhibits a jump that $\phi$ does not exhibit and, necessarily,

$$
\begin{aligned}
\phi^{\mathrm{r}}\left(t_{j+1}, j+1\right) & =\phi\left(t_{j+1}, \rho(j+1)\right)=\phi\left(t_{j+1}, \rho(j)\right) \\
& =\phi^{\mathrm{r}}\left(t_{j+1}, j\right)
\end{aligned}
$$

namely, the jump is trivial. Therefore, as long as it is defined, $\phi^{\mathrm{r}}$ is the "same" as $\phi$, modulo additional trivial jumps. If the whole hybrid $\operatorname{arc} \phi$ is "contained" in $\phi^{\mathrm{r}}$, then the parametrization is "full".

Extending the definition given in [21] for solutions to hybrid systems with inputs that share the same time domain as the state, [16, Definition 4] defines solutions to $\hat{\mathcal{H}}$ as pairs $\phi=\left(z, y^{\mathrm{r}}\right)$ where $y^{\mathrm{r}}$ is a $j$ reparametrization of $y$ that is defined on the same domain as $z$. An algorithm to build $\phi$ is provided in [16] and can be summed up as the following. As long as the input $y$ does not jump, $z$ evolves like in a standard hybrid system, $z$ flowing according to $F$ if $\phi$ is in $\hat{C}$, and jumping according to $G$ if $\phi$ is in $\hat{D}$. In this case, a trivial jump is added to $y^{\mathrm{r}}$. On the other hand, when $y$ jumps, $z$ can either jump according to $G$ or be reset identically, depending on whether $\phi$ is in $\hat{C}, \hat{D}$ or both. The precise jump logic is recalled in Appendix. 


\subsection{Definition of Asymptotic Observer for $\mathcal{H}$}

Definition 2.2. Let $\mathcal{A}$ be a nonempty subset of $\mathbb{R}^{d_{x}} \times \mathbb{R}^{d_{x}}$. The hybrid system $\hat{\mathcal{H}}$ is an asymptotic observer for $\mathcal{H}$ on $\mathcal{X}_{0} \subseteq \mathbb{R}^{d_{x}}$ relative to $\mathcal{A}$ if there exist a distance function $d_{\mathcal{A}}$ relative to $\mathcal{A}$ and a subset $\mathcal{Z}_{0}$ of $\mathbb{R}^{d_{z}}$ such that for any complete plant solution $x \in \mathcal{S}_{\mathcal{H}}\left(\mathcal{X}_{0}\right)$ with output $y$ and any observer solution $\phi=\left(z, y^{\mathrm{r}}\right) \in \mathcal{S}_{\hat{\mathcal{H}}}\left(\mathcal{Z}_{0} ; y\right)$ with output $\hat{x}$ and $j$-reparametrization map $\rho$ from $y$ to $y^{\mathrm{r}}$ :

(a) $\phi$ is complete with $\operatorname{dom}_{t} \phi=\operatorname{dom}_{t} x$;

(b) denoting $x^{\mathrm{r}}$ the full $j$-reparametrization of $x$ on the domain of $\phi$ defined by

$$
x^{\mathrm{r}}(t, j)=x(t, \rho(j)) \quad \forall(t, j) \in \operatorname{dom} \phi,
$$

we have

$$
\lim _{t+j \rightarrow+\infty} d_{\mathcal{A}}\left(\hat{x}(t, j), x^{\mathrm{r}}(t, j)\right)=0 .
$$

When $\mathcal{A}$ is the diagonal set (5), we just say "asymptotic observer for $\mathcal{H}$ on $\mathcal{X}_{0}$ ".

Condition (a) ensures that the observer solution exists as long as the underlying plant solution $x$ does. In particular, the extra condition $\operatorname{dom}_{t} x=\operatorname{dom}_{t} \phi$ means that they both "achieve their completeness" at the same time:

- either they are both $t$-complete;

- or they are both Zeno with same $\operatorname{dom}_{t}$.

As for Condition (b), it translates the intuitive idea of " $\hat{x}$ converges to $x$ " (relative to $\mathcal{A}$ ), even if $\hat{x}$ and $x$ do not share the same domain. This is done by reparametrizing $x$ into $x^{\mathrm{r}}$, which is defined on the domain of $\hat{x}$.

When convergence of $\hat{x}$ to $x$ is required, $\mathcal{A}$ can be chosen as the diagonal set (5). However, the generic set $\mathcal{A}$ in Definition 2.2 allows to consider more general notions of convergence of $(x, \hat{x})$. This is important because exact convergence of $\hat{x}$ to $x$ is in general difficult to obtain unless $g=$ Id or unless the jumps of the observer become perfectly synchronized with those of the plant after some time. Indeed, if $\hat{x}$ and $x$ do not jump exactly at the same time and $g \neq \mathrm{Id}$, it may not be possible to make the estimation error $\hat{x}-x$ small: if $x=\hat{x}$ before the jump, then $\hat{x} \in g(x) \cup g^{-1}(x)$ after one jump of either $x$ or $\hat{x}$. This is the so-called peaking phenomenon. In that case, denoting

$$
\underline{g}(x)=\left\{\begin{array}{ll}
g(x) & \text { if } x \in D \\
\emptyset & \text { otherwise }
\end{array},\right.
$$

we can only expect $(x, \hat{x})$ to converge to

$$
\mathcal{A}=\left\{(x, \hat{x}) \in(C \cup D \cup g(D))^{2}: x=\hat{x} \quad \text { or } x \in \underline{g}(\hat{x}) \text { or } \hat{x} \in \underline{g}(x)\right\},
$$

as in [6], or even

$$
\mathcal{A}=\left\{(x, \hat{x}) \in(C \cup D \cup g(D))^{2}: \exists k \in \mathbb{N}: x \in \underline{g}^{k}(\hat{x}) \text { or } \hat{x} \in \underline{g}^{k}(x)\right\}
$$

when consecutive jumps are possible [17]. More generally, we might be interested in estimating only part of the state $x$, which can be captured by a proper choice of $\mathcal{A}$.

\section{Hybrid Asymptotic Detectability}

In order to define detectability in a way that extends Definition 1.2, we need to compare the outputs of two hybrid solutions and decide whether they are "equal" in some sense. 


\subsection{Motivation}

Methods to compare hybrid arcs have been developed in the literature. In [20, Definition 5.23], notions of $\varepsilon$ and $(\varepsilon, \tau)$-closeness were first introduced. This distance is related to the graphical distance of the graphs of the hybrid arcs, namely hybrid arcs are compared at the same jump index and "close in time." It was used in the context of incremental stability [22], but was then observed to be too restrictive [18] and was consequently relaxed in [18, Definition 4] or in [19, Definition 1] by allowing to compare solutions "close in time" but maybe at different jump indexes.

In the context of detectability, we must decide under which conditions on the outputs we want to require trajectories to converge to each other. Our approach is to think of detectability as a necessary condition for the existence of an observer, similarly to CT and DT systems. Therefore, the question becomes: which pairs of outputs would the observer in Definition 2.2 not distinguish? The observer would then produce asymptotically the same estimate and the corresponding plant trajectories would thus have to converge to each other, along a similar reasoning as in Theorem 1.3.

First, we notice that Definition 2.2 concerns only complete trajectories and that the estimate $\hat{x}$ is also complete with the same time horizon. Therefore, in comparing pairs of complete solutions $\left(x_{a}, x_{b}\right)$ of $\mathcal{H}$ with the "same output", the only way we can exploit the observer asymptotic convergence is if $\operatorname{dom}_{t} x_{a}=\operatorname{dom}_{t} x_{b}$.

The meaning of "same output" should then be clarified. In the spirit of graphical distance [20], equality of outputs would require equality of the time domains. Of course, if two plant trajectories have the same domain and same output, they will produce in the observer the same trajectories $\hat{x}$ and should be asked to converge to each other. This is however restrictive because the observer may not either be able to distinguish outputs that are the same up to trivial jumps added to their domains. On the other hand, the spirit of [18, Definition 4] would consider two outputs $y_{a}$ and $y_{b}$ "equal" if for all $(t, j) \in \operatorname{dom} y_{a}$,

$$
\exists j^{\prime} \in \mathbb{N}:\left(t, j^{\prime}\right) \in \operatorname{dom} y_{b}, y_{a}(t, j)=y_{b}\left(t, j^{\prime}\right)
$$

and vice-versa. This time, this definition would be too broad since it does not respect the causality/order of the jumps which indeed is seen by the observer. In particular, this definition would not apply to DT systems.

All in all, we propose an intermediate definition based on an algorithm that reparametrizes two hybrid arcs onto a common time domain in order to compare them pointwisely, while preserving the order and simultaneity of the jumps.

\subsection{Algorithm $\mathcal{R}_{c}$}

Two hybrid $\operatorname{arcs} x_{a}$ and $x_{b}$ can be reparametrized onto a common hybrid time domain, constructed by either

- preserving the time stamp and, as time evolves, adding jumps whenever either $x_{a}$ or $x_{b}$ jumps. When only one hybrid arc jumps, a trivial jump is added to the other; when both jump, their jumps are recorded simultaneously;

- or preserving the jump numbering and letting both hybrid arcs flow until they can both jump at the same time. When one arc flows for a longer time than the other, the other arc is kept constant while waiting for the other's jump time.

Given the role of time in applications, we explore the first process as formalized in Algorithm 3.1. By preserving the order and simultaneity of the jumps, this process also applies to discrete hybrid arcs.

Definition 3.1. Given two hybrid arcs $x_{a}$ and $x_{b}$, we define the reparametrized hybrid arcs $\left(x_{a}^{\mathrm{r}}, x_{b}^{\mathrm{r}}\right):=$ $\mathcal{R}_{c}\left(x_{a}, x_{b}\right)$ by Algorithm 3.1. 


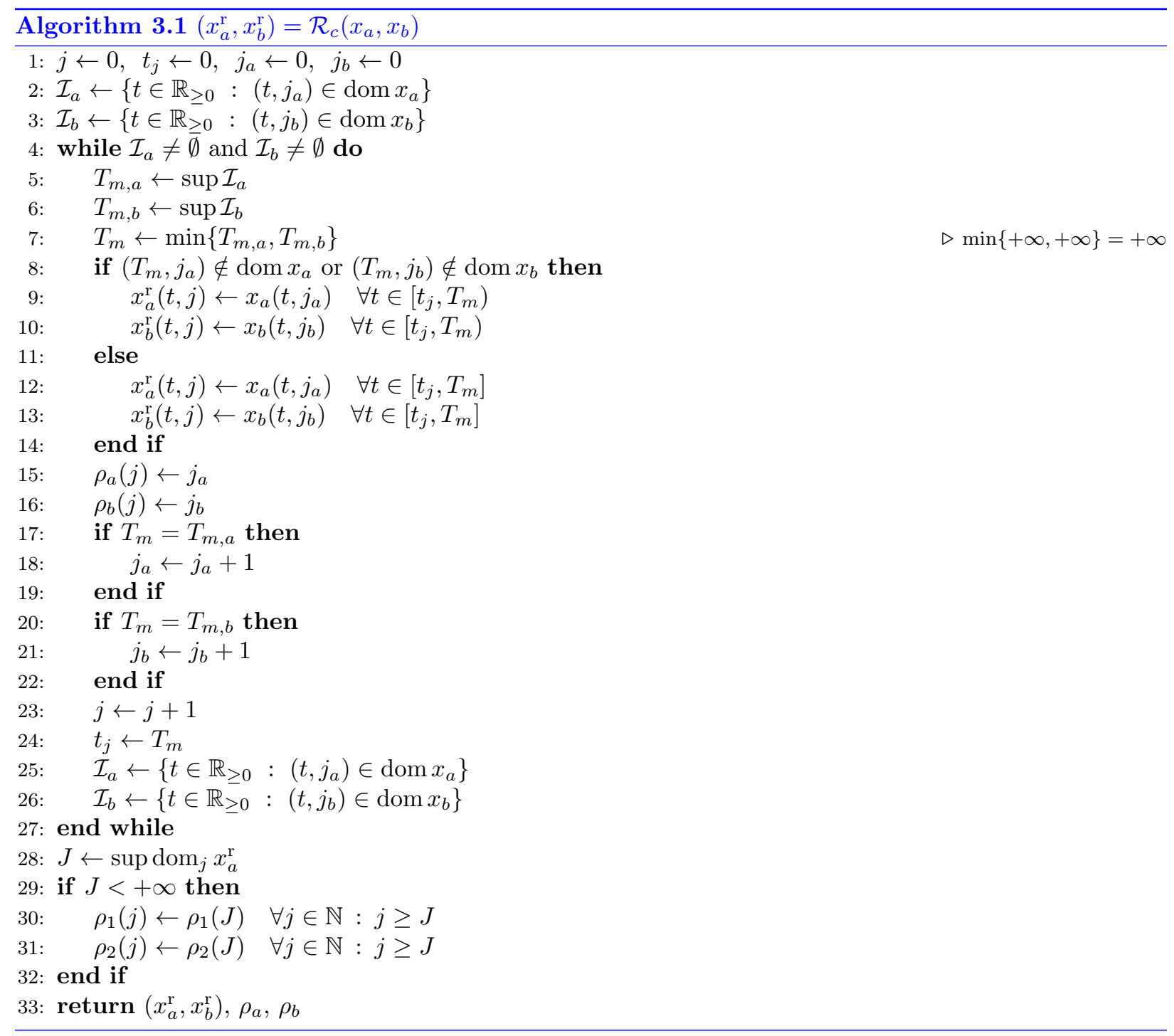


Example 3.2. Take two continuous hybrid arcs $x_{a}$ and $x_{b}$ defined on $\mathcal{D}_{a}=I_{a} \times\{0\}$ and $\mathcal{D}_{b}=I_{b} \times\{0\}$ respectively, where $I_{a}$ and $I_{b}$ are intervals of $\mathbb{R}_{\geq 0}$ containing 0 . Then, because neither $x_{a}$ nor $x_{b}$ has jumps, Algorithm 3.1 gives the output in one iteration which is $\mathcal{R}_{c}\left(x_{a}, x_{b}\right)=\left(x_{1 \mid \mathcal{D}}, x_{2 \mid \mathcal{D}}\right)$ with $\mathcal{D}=\left(I_{a} \cap I_{b}\right) \times\{0\}$. Therefore, there is no loss of information if and only if $I_{a}=I_{b}$. Otherwise, one solution is stopped by the other and the resulting hybrid arc has a domain that does not cover the original domains.

Example 3.3. Take two discrete hybrid arcs $x_{a}$ and $x_{b}$ defined on $\mathcal{D}_{a}=\{0\} \times\left\{0,1, \ldots, J_{a}\right\}$ and $\mathcal{D}_{b}=$ $\{0\} \times\left\{0,1, \ldots, J_{b}\right\}$ respectively. With Algorithm 3.1, $\mathcal{R}_{c}\left(x_{a}, x_{b}\right)=\left(x_{a \mid \mathcal{D}}, x_{b \mid \mathcal{D}}\right)$ with $\mathcal{D}=\{0\} \times\{0,1, \ldots, J=$ $\left.\min \left\{J_{a}, J_{b}\right\}\right\}$. Therefore, all the information about $x_{a}$ and $x_{b}$ is kept if only if $J_{a}=J_{b}$.

Example 3.4. Take a continuous hybrid arc $x_{a}$ defined on $\mathcal{D}_{a}=I_{a} \times\{0\}$ and a discrete hybrid arc $x_{b}$ defined on $\mathcal{D}_{b}=\{0\} \times\left\{0,1, \ldots, J_{b}\right\}$. Since Algorithm 3.1 completes all the jumps at a given time before moving further in time, $\mathcal{R}_{c}\left(x_{a}, x_{b}\right)=\left(x_{a}^{\mathrm{r}}, x_{b}^{\mathrm{r}}\right)$ is defined on $\mathcal{D}=\{0\} \times\left\{0,1, \ldots, J_{b}\right\}=\mathcal{D}_{b}$ by

$$
x_{a}^{\mathrm{r}}(0, j)=x_{a}(0,0) \quad \forall j \in\left\{0,1, \ldots, J_{b}\right\} \quad, \quad x_{b}^{\mathrm{r}} \equiv x_{b}
$$

so that $x_{a}^{\mathrm{r}}$ is blocked to its initial value.

We see from those examples that Algorithm 3.1 preserves the time stamp, but changes the jump numbering, and it stops when one of the arcs has reached the end of its domain. It thus gives pairs $\left(x_{a}^{\mathrm{r}}, x_{b}^{\mathrm{r}}\right)$ defined on a common time domain which are $j$-reparametrizations of $x_{a}$ and $x_{b}$, at least on the "common" part of their domains. However, it can happen that $x_{a}$ ends earlier and "blocks" $x_{b}$ so that $x_{b}^{\mathrm{r}}$ does not contain all the information about $x_{b}$, i.e., it is not a full $j$-reparametrization.

Lemma 3.5. Consider two complete hybrid arcs $x_{a}$ and $x_{b}$ such that $\operatorname{dom}_{t} x_{a}=\operatorname{dom}_{t} x_{b}$. Then, the hybrid $\operatorname{arc}\left(x_{a}^{\mathrm{r}}, x_{b}^{\mathrm{r}}\right)=\mathcal{R}_{c}\left(x_{a}, x_{b}\right)$ is such that both $x_{a}^{\mathrm{r}}$ and $x_{b}^{\mathrm{r}}$ are full $j$-reparametrizations of $x_{a}$ and $x_{b}$, respectively.

Proof Let's denote $\phi^{\mathrm{r}}=\left(x_{a}^{\mathrm{r}}, x_{b}^{\mathrm{r}}\right)$. First note that Algorithm 3.1 browses in time the domains of $x_{a}$ and $x_{b}$ until at least one of them has been completely browsed, i.e. when $\mathcal{I}_{a}$ or $\mathcal{I}_{b}$ therein becomes empty. This means that $\operatorname{dom}_{t} \phi^{\mathrm{r}}=\operatorname{dom}_{t} x_{a} \cap \operatorname{dom}_{t} x_{b}=\operatorname{dom}_{t} x_{a}$. Besides, a jump occurs in $\phi^{\mathrm{r}}$ only at times in $\operatorname{dom}_{t} \phi^{\mathrm{r}}$ where either $x_{a}$ or $x_{b}$ jumps. At such a time $t_{j}, \phi^{\mathrm{r}}$ jumps as long as both $x_{a}$ and $x_{b}$ jump. Assume $x_{a}$ stops jumping before $x_{b}$. Then, either $x_{a}$ flows for $t>t_{j}$ or $x_{a}$ stops.

- In the former case, $\mathcal{I}_{a}$ contains the next interval of flow, $T_{m} \neq T_{m, a}, j_{b}$ stays constant, $T_{m}$ remains equal to $t_{j}$ and $\phi^{\mathrm{r}}$ carries on jumping as long as $x_{b}$ jumps.

- in the latter case, $\mathcal{I}_{a}$ is empty and $\phi^{\mathrm{r}}$ stops.

In other words,

1. $\operatorname{dom}_{t} \phi^{\mathrm{r}}=\operatorname{dom}_{t} x_{a} \cap \operatorname{dom}_{t} x_{b}$, so that $T^{\mathrm{r}}:=T\left(\phi^{\mathrm{r}}\right)=\min \left\{T\left(x_{a}\right), T\left(x_{b}\right)\right\}$

2. $\operatorname{card} \mathcal{J}^{t}\left(\phi^{\mathrm{r}}\right)=\max \left\{\operatorname{card} \mathcal{J}^{t}\left(x_{a}\right)\right.$, card $\left.\mathcal{J}^{t}\left(x_{b}\right)\right\}$ for all $t \in \operatorname{dom}_{t} \phi^{\mathrm{r}} \backslash\left\{T^{\mathrm{r}}\right\}$

3. $\operatorname{card} \mathcal{J}^{T^{\mathrm{r}}}\left(\phi^{\mathrm{r}}\right)= \begin{cases}\operatorname{card} \mathcal{J}^{T^{\mathrm{r}}}\left(x_{a}\right) & \text { if } T\left(x_{b}\right)>T^{\mathrm{r}} \\ \operatorname{card} \mathcal{J}^{T^{\mathrm{r}}}\left(x_{b}\right) & \text { if } T\left(x_{a}\right)>T^{\mathrm{r}} \\ \min \left\{\operatorname{card} \mathcal{J}^{T^{\mathrm{r}}}\left(x_{a}\right), \operatorname{card} \mathcal{J}^{T^{\mathrm{r}}}\left(x_{b}\right)\right\} & \text { if } T\left(x_{a}\right)=T\left(x_{b}\right)=T^{\mathrm{r}}\end{cases}$

Consider now the functions $\rho_{a}: \mathbb{N} \rightarrow \mathbb{N}$ and $\rho_{b}: \mathbb{N} \rightarrow \mathbb{N}$ built in Algorithm 3.1. They clearly verify (8) and because they record $j_{a}$ and $j_{b}$ respectively, we have

$$
x_{a}^{\mathrm{r}}(t, j)=x_{a}\left(t, \rho_{a}(j)\right) \quad \forall(t, j) \in \operatorname{dom} x_{a}^{\mathrm{r}} \quad, \quad x_{b}^{\mathrm{r}}(t, j)=x_{b}\left(t, \rho_{b}(j)\right) \quad \forall(t, j) \in \operatorname{dom} x_{b}^{\mathrm{r}}
$$

This exactly means that $x_{a}^{\mathrm{r}}$ and $x_{b}^{\mathrm{r}}$ are $j$-reparametrizations of $x_{a}$ and $x_{b}$.

Now assume $x_{a}$ and $x_{b}$ are complete and $\operatorname{dom}_{t} x_{a}=\operatorname{dom}_{t} x_{b}$. Then, items 1,2,3 above imply that those parametrizations are full. 


\subsection{A Definition of Asymptotic Detectability}

The following definition extends Definition 1.2.

Definition 3.6. Let $\mathcal{A}$ be a nonempty subset of $\mathbb{R}^{d_{x}} \times \mathbb{R}^{d_{x}}$. The hybrid plant $\mathcal{H}$ is said to be asymptotically detectable on $\mathcal{X}_{0}$ relative to $\mathcal{A}$ if there exists a distance function $d_{\mathcal{A}}$ relative to $\mathcal{A}$ such that any pair of complete solutions $x_{a}, x_{b} \in \mathcal{S}_{\mathcal{H}}\left(\mathcal{X}_{0}\right)$ verifying $\operatorname{dom}_{t} x_{a}=\operatorname{dom}_{t} x_{b}$ and

$$
h\left(x_{a}^{\mathrm{r}}(t, j)\right)=h\left(x_{b}^{\mathrm{r}}(t, j)\right) \quad \forall(t, j) \in \operatorname{dom} \phi^{\mathrm{r}}
$$

where $\left(x_{a}^{\mathrm{r}}, x_{b}^{\mathrm{r}}\right):=\mathcal{R}_{c}\left(x_{a}, x_{b}\right)$, verify

$$
\lim _{t+j \rightarrow+\infty} d_{\mathcal{A}}\left(x_{a}^{\mathrm{r}}(t, j), x_{b}^{\mathrm{r}}(t, j)\right)=0 .
$$

Note that the mention of $\mathcal{A}$ can be omitted if it is simply the diagonal set defined in (5).

Asymptotic detectability requires that any two solutions which are complete on the same "time horizon" and have the "same" output (once put on a same domain via $\mathcal{R}_{c}$ ), asymptotically converge to each other (in the sense of $\mathcal{A}$ ). This definition enables to recover the standard notions of asymptotic detectability of CT and DT systems, since in those cases, $x_{a}=x_{a}^{\mathrm{r}}$ and $x_{b}=x_{b}^{\mathrm{r}}$.

\section{Observers and Detectability via Extended Systems}

Before proceeding to the proof of Theorem 1.5, we exhibit the link between our definitions and alternative definitions via extended systems, which will be useful for the proof.

\subsection{Observers}

Instead of defining an observer as $\hat{\mathcal{H}}$ in (7), a first idea could have been to define an observer directly through an extended system of the form

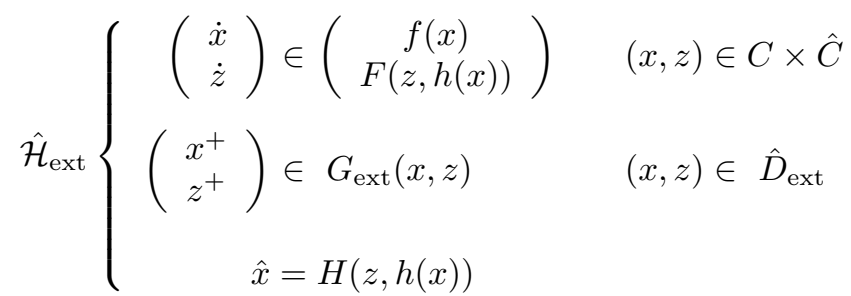

with a jump map $G_{\text {ext }}$ and a jump set $\hat{D}_{\text {ext }}$ to be defined. In doing that, we are facing three main difficulties. First, a jump logic has to be designed in a way that does not assume synchronous jumps of $z$ and $x$ since the jump times of the plant $\mathcal{H}$ are not necessarily known. Second, it is not straightforward to deduce from $\hat{\mathcal{H}}_{\text {ext }}$ the hybrid dynamics of $z$ to be implemented as an observer algorithm with input $y$ and output $\hat{x}$. Third, without any assumption about the domain of solutions to $\mathcal{H}$, we would require something like: any complete solution of $\hat{\mathcal{H}}_{\text {ext }}$ initialized in $\mathcal{X}_{0} \times \mathcal{Z}_{0}$ verifies $\lim _{t+j \rightarrow+\infty} d_{\mathcal{A}}(x(t, j), \hat{x}(t, j))=0$. But a solution to $\hat{\mathcal{H}}_{\text {ext }}$ may be complete without browsing the whole underlying maximal solution of $\mathcal{H}$, for instance if the $z$-component induced Zeno or finite time escape earlier than $x$. Therefore, $\hat{x}$ would not provide any estimate of $x$ after a certain time, which is not acceptable.

This being said, an extended system of the form (16) may be handy for design since it allows for Lyapunov analysis. Actually, in [23, Section 4.1], solutions $\left(z, y^{\mathrm{r}}\right)$ to $\hat{\mathcal{H}}$ are shown to be such that $\left(x^{\mathrm{r}}, z\right)$ is solution to $\hat{\mathcal{H}}_{\text {ext }}$ with jump set

$$
\begin{aligned}
\hat{D}_{\text {ext }}=\left\{(x, z) \in \mathbb{R}^{d_{x}} \times \mathbb{R}^{d_{z}}: x \in D,(z, h(x)) \in \operatorname{cl}(\hat{C}) \cup \hat{D}\right\} & \\
& \cup\left\{(x, z) \in \mathbb{R}^{d_{x}} \times \mathbb{R}^{d_{z}}: x \in \operatorname{cl}(C) \cup D,(z, h(x)) \in \hat{D}\right\}
\end{aligned}
$$


and jump map

$$
G_{\text {ext }}(x, z)=\left(\begin{array}{c}
g(x) \\
\underline{\operatorname{Id}}_{z}(z, h(x))
\end{array}\right) \cup\left(\begin{array}{c}
\underline{\mathrm{Id}}_{x}(x) \\
\underline{G}(z, h(x))
\end{array}\right) \cup\left(\begin{array}{c}
g \\
\underline{G}(x) \\
\underline{z}, h(x))
\end{array}\right)
$$

where $g$ is defined in (12), and in the same spirit

$$
\begin{gathered}
\underline{G}(z, h(x))=\left\{\begin{array}{ll}
G(z, h(x)) & \text { if }(z, h(x)) \in \hat{D} \\
\emptyset & \text { otherwise }
\end{array},\right. \\
\underline{\operatorname{Id}}_{x}(x)=\left\{\begin{array}{ll}
x & \text { if } x \in \operatorname{cl}(C) \\
\emptyset & \text { otherwise }
\end{array},\right. \\
\underline{\operatorname{Id}}_{z}(z, h(x))=\left\{\begin{array}{ll}
z & \text { if }(z, h(x)) \in \operatorname{cl}(\hat{C}) \\
\emptyset & \text { otherwise }
\end{array} .\right.
\end{gathered}
$$

Therefore, any analysis made on $\hat{\mathcal{H}}_{\text {ext }}$ may hold for solutions of $\hat{\mathcal{H}}$. However, the reverse is not true because $\hat{\mathcal{H}}_{\text {ext }}$ has a larger set of solutions, see [16] for more details.

Lemma 4.1. Let $\mathcal{A}$ be a nonempty subset of $\mathbb{R}^{d_{x}} \times \mathbb{R}^{d_{x}}$ and $d_{\mathcal{A}}$ a distance relative to $\mathcal{A}$. Assume any $x \in \mathcal{S}_{\mathcal{H}}\left(\mathcal{X}_{0}\right)$ is t-complete. If each $(x, z) \in \mathcal{S}_{\hat{\mathcal{H}}_{\text {ext }}}\left(\mathcal{X}_{0} \times \mathcal{Z}_{0}\right)$ is t-complete and verifies

$$
\lim _{t+j \rightarrow+\infty} d_{\mathcal{A}}(x(t, j), \hat{x}(t, j))=0,
$$

then $\hat{\mathcal{H}}$ is an asymptotic observer for $\mathcal{H}$ on $\mathcal{X}_{0}$ relative to $\mathcal{A}$.

Proof Consider $x \in \mathcal{S}_{\mathcal{H}}\left(\mathcal{X}_{0}\right)$ and $\phi=\left(z, y^{\mathrm{r}}\right) \in \mathcal{S}_{\hat{\mathcal{H}}}\left(\mathcal{Z}_{0} ; h(x)\right)$. According to [23, Lemma 1], $\left(x^{\mathrm{r}}, z\right) \in$ $\mathcal{S}_{\hat{\mathcal{H}}_{\text {ext }}}\left(\mathcal{X}_{0} \times \mathcal{Z}_{0}\right)$. Therefore, $\phi$ is $t$-complete like $x$, and both items (a) and (b) of Definition 2.2 hold.

Remark 4.2. Similar extended systems have been introduced in the literature whenever it was needed to compare hybrid arcs with different domains, for instance in the context of reference tracking [17] or incremental stability [18]. The main difference is that we allow here both $x$ and $z$ to jump simultaneously with $g$ and $G$, whereas in $[17,18]$ this kind of jump is decomposed into two successive jumps. The main reason for allowing simultaneous jumps here is that we want to recover the framework of DT systems when $C=\hat{C}=\emptyset$. Then, thanks to the "simultaneous jump" part of $G_{\mathrm{ext}}$, it is sufficient to allow trivial jumps only on the flow sets, as can be seen on the definition of $\mathrm{Id}_{i}$. In other words, unlike in [18], $x$ (resp. $z$ ) is forced to jump with $g$ (resp. $G$ ) on $D \backslash \operatorname{cl}(C)$ (resp. $\hat{D} \backslash \operatorname{cl}(\hat{C})$ ). Note that it is however not possible to replace $\operatorname{cl}(C)$ by $C$ in the definition of Id since $x$ could flow from $\partial C$ at a time where $z$ needs to jump, in which case a trivial jump of $x$ should be allowed (and vice-versa).

\subsection{Detectability}

Similarly to $\hat{\mathcal{H}}_{\text {ext }}$, it can be proved that given $x_{a}, x_{b} \in \mathcal{S}_{\mathcal{H}}\left(\mathcal{X}_{0}\right),\left(x_{a}^{\mathrm{r}}, x_{b}^{\mathrm{r}}\right):=\mathcal{R}_{c}\left(x_{a}, x_{b}\right)$ is a solution to the extended hybrid system

$$
\mathcal{H}^{\mathrm{r}}\left\{\begin{array}{cl}
\left(\begin{array}{c}
\dot{x}_{a} \\
\dot{x}_{b}
\end{array}\right) \in\left(\begin{array}{c}
f\left(x_{a}\right) \\
f\left(x_{b}\right)
\end{array}\right) & \left(x_{a}, x_{b}\right) \in C \times C \\
\left(\begin{array}{c}
x_{a}^{+} \\
x_{b}^{+}
\end{array}\right) \in g^{\mathrm{r}}\left(x_{a}, x_{b}\right) & \left(x_{a}, x_{b}\right) \in D^{\mathrm{r}}
\end{array}\right.
$$

where

$$
\begin{aligned}
D^{\mathrm{r}}=\left\{\left(x_{a}, x_{b}\right) \in \mathbb{R}^{d_{x}} \times \mathbb{R}^{d_{x}}: x_{a} \in D, x_{b} \in \operatorname{cl}(C) \cup D\right\} & \\
& \cup\left\{\left(x_{a}, x_{b}\right) \in \mathbb{R}^{d_{x}} \times \mathbb{R}^{d_{x}}: x_{a} \in \operatorname{cl}(C) \cup D, x_{b} \in D\right\}
\end{aligned}
$$




$$
g^{\mathrm{r}}\left(x_{a}, x_{b}\right)=\left(\begin{array}{c}
\underline{g}\left(x_{a}\right) \\
\left.\underline{\underline{\mathrm{d}}}_{x}\left(x_{b}\right)\right)
\end{array}\right) \cup\left(\begin{array}{c}
\underline{\operatorname{Id}}_{x}\left(x_{a}\right) \\
\underline{g}\left(x_{b}\right)
\end{array}\right) \cup\left(\begin{array}{c}
g\left(x_{a}\right) \\
\left.\underline{g}\left(x_{b}\right)\right)
\end{array}\right)
$$

with $\underline{g}$ and $\underline{I d}$ defined in (12) and (17). The process of duplicating $\mathcal{H}$ into $\mathcal{H}^{\mathrm{r}}$ will be denoted $\mathcal{H}^{\mathrm{r}}=: \mathcal{R}_{c}(\mathcal{H}, \mathcal{H})$.

Lemma 4.3. Let $\mathcal{A}$ be a nonempty subset of $\mathbb{R}^{d_{x}} \times \mathbb{R}^{d_{x}}$ and $d_{\mathcal{A}}$ a distance relative to $\mathcal{A}$. If each complete solution $\phi=\left(x_{a}, x_{b}\right) \in \mathcal{S}_{\mathcal{H}^{\mathrm{r}}}\left(\mathcal{X}_{0} \times \mathcal{X}_{0}\right)$ such that

$$
h\left(x_{a}(t, j)\right)=h\left(x_{b}(t, j)\right) \quad \forall(t, j) \in \operatorname{dom} \phi
$$

verifies

$$
\lim _{t+j \rightarrow+\infty} d_{\mathcal{A}}\left(x_{a}(t, j), x_{b}(t, j)\right)=0
$$

then $\mathcal{H}$ is asymptotically detectable on $\mathcal{X}_{0}$ relative to $\mathcal{A}$.

Note that this handy condition for detectability is sufficient but not necessary. Indeed, unless trajectories are $t$-complete, a complete trajectory of $\mathcal{H}^{\mathrm{r}}$ could correspond to $\mathcal{R}_{c}\left(x_{a}, x_{b}\right)$ with $x_{a}$ and $x_{b}$ not verifying $\operatorname{dom}_{t} x_{a}=\operatorname{dom}_{t} x_{b}$, which are not required to converge to each other in Definition 3.6, nor for the existence of an observer.

More generally, even if the trajectories are $t$-complete, the jump map $g^{\mathrm{r}}$ allows $x_{a}$ and $x_{b}$ to jump consecutively using first $\left(\begin{array}{c}g\left(x_{a}\right) \\ x_{b}\end{array}\right)$ and then $\left(\begin{array}{c}x_{a} \\ g\left(x_{b}\right)\end{array}\right)$ whenever $x_{a}$ and $x_{b}$ are in $D \cap \operatorname{cl}(C)$, whereas $x_{a}$ and $x_{b}$ solutions to $\mathcal{H}$ are forced to jump from $D \cap \operatorname{cl}(C)$ if no flow is possible from there. In that case, this jump would be recorded simultaneously in $\mathcal{R}_{c}\left(x_{a}, x_{b}\right)$. Therefore, $\mathcal{H}$ could be detectable relative to $\mathcal{A}$ defined in (5) without $\mathcal{H}^{\mathrm{r}}$ verifying the assumption of Lemma 4.3. Both properties are thus not equivalent. Note that they could become equivalent when delays in jumps do not matter in $\mathcal{A}$, namely for instance $\mathcal{A}$ defined in (13).

\section{Proof of Theorem 1.5}

The proof follows the same ideas as those in the proof of Theorem 1.3, but requires extra technical steps to take care of the different hybrid time domains.

Consider complete solutions $\left(x_{a}, x_{b}\right) \in \mathcal{S}_{\mathcal{H}}\left(\mathcal{X}_{0}\right) \times \mathcal{S}_{\mathcal{H}}\left(\mathcal{X}_{0}\right)$ with $\mathcal{H}$ in $(4)$ such that $\operatorname{dom}_{t} x_{a}=\operatorname{dom}_{t} x_{b}$ and $\phi^{\mathrm{r}}:=\left(x_{a}^{\mathrm{r}}, x_{b}^{\mathrm{r}}\right)=\mathcal{R}_{c}\left(x_{a}, x_{b}\right)$ verifies

$$
h\left(x_{a}^{\mathrm{r}}(t, j)\right)=h\left(x_{b}^{\mathrm{r}}(t, j)\right) \quad \forall(t, j) \in \operatorname{dom} \phi^{\mathrm{r}} .
$$

According to Lemma 3.5, $x_{a}^{\mathrm{r}}$ and $x_{b}^{\mathrm{r}}$ are full $j$-reparametrizations of $x_{a}$ and $x_{b}$. We denote $T:=T\left(x_{a}\right)=$ $T\left(x_{b}\right)$.

\subsection{Solution $\phi_{b}$ to $\hat{\mathcal{H}}$ with input $y_{b}=h\left(x_{b}\right)$}

Consider a maximal solution $\phi_{b}=\left(z_{b}, y_{b, \mathrm{cl}}\right)$ to $\hat{\mathcal{H}}$ in $(7)$ with $z_{b}(0,0) \in \mathcal{Z}_{0}$ and input $y_{b}=h\left(x_{b}\right)$ and denote $\rho_{b}$ the $j$-reparametrization map from $y_{b}$ to $y_{b, c l}$, which is full by Definition 2.2 and such that $\operatorname{dom}_{t} x_{b}=\operatorname{dom}_{t} \phi_{b}$. Define the corresponding full $j$-reparametrization of $x_{b}$ with

$$
x_{b, \mathrm{cl}}(t, j)=x_{b}\left(t, \rho_{b}(j)\right) \quad \forall(t, j) \in \operatorname{dom} \phi_{b}
$$

which is such that

$$
y_{b, \mathrm{cl}}(t, j)=h\left(x_{b, \mathrm{cl}}(t, j)\right) \quad \forall(t, j) \in \operatorname{dom} \phi_{b} .
$$




\subsection{From $\phi_{b}$ solution to $\hat{\mathcal{H}}$ to $\phi_{b, \mathrm{cl}}$ solution to $\hat{\mathcal{H}}_{\mathrm{ext}}$}

From [16, Lemma 1], $\phi_{b, \mathrm{cl}}=\left(x_{b, \mathrm{cl}}, z_{b}\right) \in \mathcal{S}_{\hat{\mathcal{H}}_{\text {ext }}}\left(\mathcal{X}_{0} \times \mathcal{Z}_{0}\right)$ and satisfies all the so-called Converse Conditions. At this point we have $\operatorname{dom}_{t} x_{a}=\operatorname{dom}_{t} x_{b}=\operatorname{dom}_{t} \phi_{b}=\operatorname{dom}_{t} \phi_{b, \mathrm{cl}}$ and all the hybrid arcs are complete. Also, by definition of asymptotic observer,

$$
\lim _{t+j \rightarrow+\infty} d_{\mathcal{A}}\left(\hat{x}_{b}(t, j), x_{b, \mathrm{cl}}(t, j)\right)=0
$$

with

$$
\hat{x}_{b}(t, j)=H\left(z_{b}(t, j), h\left(x_{b, \mathrm{cl}}(t, j)\right)\right) \quad \forall(t, j) \in \operatorname{dom} \phi_{b, \mathrm{cl}} .
$$

\subsection{Putting $x_{a}$ and $\phi_{b, \mathrm{cl}}$ on a common domain : construction of $\bar{\phi}$}

Consider now $\bar{\phi}=\left(\bar{x}_{a},\left(\bar{x}_{b}, \bar{z}\right)\right)=\mathcal{R}_{c}\left(x_{a},\left(x_{b, \mathrm{cl}}, z_{b}\right)\right)$. According to Lemma $3.5, \bar{x}_{a}$ and $\left(\bar{x}_{b}, \bar{z}\right)$ are full $j$-reparametrizations of $x_{a}$ and $\left(x_{b, \mathrm{cl}}, z_{b}\right)$ respectively, so $\bar{\phi}$ is complete, $\operatorname{dom}_{t} \bar{\phi}=\operatorname{dom}_{t} x_{a}$, and there exist full reparametrization maps $\rho_{a}$ and $\rho_{b, c l}: \mathbb{N} \rightarrow \mathbb{N}$ such that

$$
\bar{x}_{a}(t, j)=x_{a}\left(t, \rho_{a}(j)\right) \quad \forall(t, j) \in \operatorname{dom} \bar{\phi}
$$

and

$$
\begin{aligned}
\bar{x}_{b}(t, j) & =x_{b, \mathrm{cl}}\left(t, \rho_{b, \mathrm{cl}}(j)\right) \\
\bar{z}(t, j) & =z_{b}\left(t, \rho_{b, \mathrm{cl}}(j)\right) \quad \forall(t, j) \in \operatorname{dom} \bar{\phi}
\end{aligned}
$$

Also, since it is a full $j$-reparametrization,

$$
\lim _{t+j \rightarrow+\infty} d_{\mathcal{A}}\left(\overline{\hat{x}}(t, j), \bar{x}_{b}(t, j)\right)=0
$$

with

$$
\overline{\hat{x}}(t, j)=H\left(\bar{z}(t, j), h\left(\bar{x}_{b}(t, j)\right)\right) \quad \forall(t, j) \in \operatorname{dom} \bar{\phi} .
$$

Now, since $\operatorname{dom} x_{b, \mathrm{cl}}=\operatorname{dom} z_{b},\left(\bar{x}_{a}, \bar{x}_{b}\right)=\mathcal{R}_{c}\left(x_{a}, x_{b, \mathrm{cl}}\right)$. Since $x_{b, \mathrm{cl}}$ is a full $j$-reparametrization of $x_{b}$ processing the jumps of $x_{b}$ consecutively, according to Lemma Appendix B.2, $\left(\bar{x}_{a}, \bar{x}_{b}\right)$ is actually a full $j$-parametrization of $\phi^{\mathrm{r}}=\left(x_{a}^{\mathrm{r}}, x_{b}^{\mathrm{r}}\right)=\mathcal{R}_{c}\left(x_{a}, x_{b}\right)$, i.e. there exists a full reparametrization map $\rho^{\mathrm{r}}: \mathbb{N} \rightarrow \mathbb{N}$ such that

$$
\begin{aligned}
\bar{x}_{a}(t, j) & =x_{a}^{\mathrm{r}}\left(t, \rho^{\mathrm{r}}(j)\right) \\
\bar{x}_{b}(t, j) & =x_{b}^{\mathrm{r}}\left(t, \rho^{\mathrm{r}}(j)\right) \quad \forall(t, j) \in \operatorname{dom} \bar{\phi}
\end{aligned}
$$

It is therefore enough to show that

$$
\lim _{t+j \rightarrow+\infty} d_{\mathcal{A}}\left(\bar{x}_{a}(t, j), \bar{x}_{b}(t, j)\right)=0
$$

to deduce

$$
\lim _{t+j \rightarrow+\infty} d_{\mathcal{A}}\left(x_{a}^{\mathrm{r}}(t, j), x_{b}^{\mathrm{r}}(t, j)\right)=0
$$

which will prove asymptotic detectability according to Definition 3.6.

For that, we are going to show that actually $\lim _{t+j \rightarrow+\infty} d_{\mathcal{A}}\left(\overline{\hat{x}}(t, j), \bar{x}_{a}(t, j)\right)=0$, and use the triangle inequality with (23) to obtain (25). To that end, it is crucial to notice that (24) implies

$$
h\left(\bar{x}_{a}(t, j)\right)=h\left(\bar{x}_{b}(t, j)\right) \quad \forall(t, j) \in \operatorname{dom} \bar{\phi} .
$$




\subsection{From $\bar{\phi}$ solution to $\mathcal{R}_{c}\left(\mathcal{H}, \hat{\mathcal{H}}_{\text {ext }}\right)$, to $\phi_{a, c l}$ solution to $\hat{\mathcal{H}}_{\text {ext }}$}

Now, according to Lemma Appendix C.3, $\bar{\phi}=\left(\bar{x}_{a},\left(\bar{x}_{b}, \bar{z}\right)\right)$ is a maximal solution to $\mathcal{R}_{c}\left(\mathcal{H}, \hat{\mathcal{H}}_{\text {ext }}\right)$ satisfying Conditions 1 and 2. But because of $(26),\left(\bar{x}_{b},\left(\bar{x}_{a}, \bar{z}\right)\right)$ is actually also a maximal solution to $\mathcal{R}_{c}\left(\mathcal{H}, \hat{\mathcal{H}}_{\text {ext }}\right)$ on $\operatorname{dom} \bar{\phi}$. At this point, we would like to recover from $\left(\bar{x}_{a}, \bar{z}\right)$ a solution to $\hat{\mathcal{H}}_{\text {ext }}$ to reuse the observer definition. For this, we need to check that $\left(\bar{x}_{a}, \bar{z}\right)$ verifies Condition 2 to apply the converse part of Lemma Appendix C.3. For all $t \in \mathcal{T}(\bar{\phi}) \cap \operatorname{int} \operatorname{dom}_{t}(\bar{\phi})$ such that $\left(\bar{x}_{a}, \bar{z}\right)$ does not verify the jump condition of $\mathcal{H}_{c l}$ for any $j \in \mathcal{J}^{t}(\bar{\phi})$, necessarily $\bar{x}_{a}$ does not verify the jump condition of $\mathcal{H}$ and $\bar{z}$ does not verify the jump condition of $\hat{\mathcal{H}}$. It means that $t \notin \mathcal{T}\left(x_{a}\right)$, and since $t \in \operatorname{int} \operatorname{dom}_{t}\left(x_{a}\right), x_{a} \in C$ by definition of solutions to $\mathcal{H}$. Therefore, those jumps are necessarily triggered by $\bar{x}_{b}$ and necessarily $\left(\bar{z}, h\left(\bar{x}_{a}\right)\right)=\left(\bar{z}, h\left(\bar{x}_{b}\right)\right) \in \hat{C}$ by item 4.(a) of Definition Appendix A.1. Therefore, $\left(\bar{x}_{a}, \bar{z}\right)$ verifies Condition 2. We thus deduce from Lemma Appendix C.3 that there exists a solution $\phi_{a, \mathrm{cl}}=\left(x_{a, \mathrm{cl}}, z_{a}\right)$ to $\hat{\mathcal{H}}_{\text {ext }}$ and a reparametrization map $\rho_{a, \mathrm{cl}}: \mathbb{N} \rightarrow \mathbb{N}$ such that

$$
\begin{aligned}
\bar{x}_{a}(t, j) & =x_{a, \mathrm{cl}}\left(t, \rho_{a, \mathrm{cl}}(j)\right) \\
\bar{z}(t, j) & =z_{a}\left(t, \rho_{a, \mathrm{cl}}(j)\right)
\end{aligned} \quad \forall(t, j) \in \operatorname{dom} \bar{\phi}
$$

and $\left(\bar{x}_{a}, \bar{z}\right)$ is a full $j$-reparametrization of $\left(x_{a, \mathrm{cl}}, z_{a}\right)$ with $\operatorname{dom}_{t} \phi_{a, \mathrm{cl}}=\operatorname{dom}_{t} \bar{\phi}$. If this time domain is unbounded, $\phi_{a, \mathrm{cl}}$ is complete. If it is bounded, because $\bar{x}_{a}$ is a full $j$-reparametrization of $x_{a}$ which is complete, the number of jumps where $\left(\bar{x}_{a}, \bar{z}\right)$ jumps according to $\hat{\mathcal{H}}_{\text {ext }}$ is infinite. Therefore, by construction $\phi_{a, \mathrm{cl}}$ is $j$-complete. So in both cases, $\phi_{a, \mathrm{cl}}$ is complete and $\operatorname{dom}_{t} \phi_{a, \mathrm{cl}}=\operatorname{dom}_{t} \bar{\phi}$.

\subsection{From $\phi_{a, c l}$ solution to $\hat{\mathcal{H}}_{\mathrm{ext}}$ to $\phi_{a}$ solution to $\hat{\mathcal{H}}$ with input $y_{a}^{\prime}$}

The last step is to come back from $\hat{\mathcal{H}}_{\text {ext }}$ to an observer solution, namely prove that there exists $x_{a}^{\prime}$ solution to $\mathcal{H}$ initialized in $\mathcal{X}_{0}$ such that $\left(z_{a}, y_{a, \mathrm{cl}}\right)$ is solution to $\hat{\mathcal{H}}$ with input $y_{a}^{\prime}=h\left(x_{a}^{\prime}\right)$ where $y_{a, \mathrm{cl}}=h\left(x_{a, \mathrm{cl}}\right)$ and $x_{a, \mathrm{cl}}$ is a full $j$-reparametrization of $x_{a}^{\prime}$. This is done by applying the converse part of [16, Lemma 1]. For that, we need to show that $\phi_{a, \mathrm{cl}}$ verifies all the so-called Converse Conditions ( ). First, $\mathcal{H}_{c l}$ has no input, so the conditions regarding $n_{u_{a}}$ don't have to be checked (in particular CC.2)). Then it is useful to recall that with the algorithm in the converse of Lemma Appendix C.3, $\phi_{a, c l}$ is obtained from $\bar{\phi}$ only by removing the jumps where $\left(\bar{x}_{a}, \bar{z}\right)$ does not verify the jump condition of $\mathcal{H}_{c l}$. The existence of $n_{x_{a}}$ verifying the CC.1) is therefore guaranteed by the fact that the jumps of $x_{a}$ where recorded successively in $\bar{x}_{a}$ thanks to condition C1. Also, at a time $t>0$ where $n_{x_{a}} \geq 1$, either $\phi_{b, c l}=\left(x_{b, c l}, z\right)$ also jumped at that time, in which case CC.3) holds for $\phi_{a, c l}$ because it holds for $\phi_{b, c l}$ and because the value of $h\left(\bar{x}_{a}\right)$ corresponds to that of $h\left(\bar{x}_{b}\right)$ at that jump; or $\phi_{b, c l}$ does not jump at that time $\left(\operatorname{card} \mathcal{J}_{t}\left(\phi_{b, c l}\right)=0\right)$ and $\left(\bar{z}, h\left(\bar{x}_{a}\right)\right)=\left(\bar{z}, h\left(\bar{x}_{b}\right)\right)$ is necessarily in $\hat{C}$ since $t>0$, so that there is nothing to check in CC.3). Therefore, CC.3) holds. Then, at a time $t$ in $\operatorname{int} \phi_{a, c l}$ where $n_{x_{a}}=0, x_{a, c l}$ is in $C$ thanks to Condition 2 verified by $\bar{x}_{a}$, thus giving CC.4). Finally, if $T \in \operatorname{dom}_{t} \phi_{a, c l}=\operatorname{dom}_{t} x_{a}$, because $x_{a}$ and $\phi_{a, c l}$ are complete, CC.5) holds (with $n_{x_{a}}=+\infty$ ). Therefore, $\phi_{a, c l}=\left(x_{a, c l}, z_{a}\right)$ verifies all the converse conditions. Now, again, either $\operatorname{dom}_{t} x_{a, \mathrm{cl}}=\operatorname{dom}_{t} x_{a}^{\prime}$ is unbounded and $x_{a}^{\prime}$ is complete. Or, $\operatorname{dom}_{j} x_{a, \mathrm{cl}}$ is unbounded and contains by construction the infinite number of jumps of $x_{a}$. Therefore, since $x_{a}^{\prime}$ is built from $x_{a, \mathrm{cl}}$ by only removing the jumps which does not verify the jump conditions of $\mathcal{H}, x_{a}^{\prime}$ has an infinite number of jumps. It follows that $x_{a}^{\prime}$ is necessarily complete. From the observer definition, it thus follows that

$$
\lim _{t+j \rightarrow+\infty} d_{\mathcal{A}}\left(\hat{x}_{a}(t, j), x_{a, \mathrm{cl}}(t, j)\right)=0
$$

where

$$
\hat{x}_{a}(t, j)=H\left(z_{a}(t, j), h\left(x_{a, \mathrm{cl}}(t, j)\right)\right) .
$$

Since $\left(\bar{x}_{a}, \bar{z}\right)$ is a full $j$-reparametrization of $\left(x_{a, \mathrm{cl}}, z_{a}\right)$, from (27) and (28)-(29), we then deduce that

$$
\lim _{t+j \rightarrow+\infty} d_{\mathcal{A}}\left(H\left(\bar{z}(t, j), h\left(\bar{x}_{a}(t, j)\right)\right), \bar{x}_{a}(t, j)\right)=0
$$


and with (26),

$$
\lim _{t+j \rightarrow+\infty} d_{\mathcal{A}}\left(\overline{\hat{x}}(t, j), \bar{x}_{a}(t, j)\right)=0
$$

By triangle inequality, using (6),

$$
d_{\mathcal{A}}\left(\bar{x}_{a}(t, j), \bar{x}_{b}(t, j)\right) \leq d_{\mathcal{A}}\left(\bar{x}_{a}(t, j), \overline{\hat{x}}(t, j)\right)+d_{\mathcal{A}}\left(\overline{\hat{x}}(t, j), \bar{x}_{b}(t, j)\right)
$$

and from (23), we finally obtain (25).

Remark 5.1. This proof, similarly to the proof of Theorem 1.3, heavily relies on a triangle inequality, so property (6c) is crucial. This differs from [18] where the distance is only required to be definite (6a) and symmetric $(6 \mathrm{~b})$.

\section{Appendix A. Definition of solutions to $\hat{\mathcal{H}}$}

Definition Appendix A.1. Consider a hybrid arc y. A pair $\phi=\left(z, y^{\mathrm{r}}\right)$ is a solution to $\hat{\mathcal{H}}$ in $(7)$ with input $y$ and output $\hat{x}$ if

1. $\operatorname{dom} z=\operatorname{dom} y^{\mathrm{r}}(=\operatorname{dom} \phi)$

2. $y^{\mathrm{r}}$ is a j-reparametrization of y with reparametrization map $\rho_{y}$, and with also card $\mathcal{J}_{T(y)}(\phi)=\operatorname{card} \mathcal{J}_{T(y)}(y)$ if this reparametrization is full.

3. for all $j \in \mathbb{N}$ such that $\mathcal{I}_{j}(\phi)$ has nonempty interior,

$$
\begin{array}{r}
\left(z(t, j), y^{\mathrm{r}}(t, j)\right) \in \hat{C} \quad \forall t \in \operatorname{int} \mathcal{I}_{j}(\phi) \\
\dot{z}(t, j) \in F\left(z(t, j), y^{\mathrm{r}}(t, j)\right) \quad \text { for almost all } t \in \mathcal{I}_{j}(\phi)
\end{array}
$$

4. for all $t \in \mathcal{T}(\phi)$, denoting $j_{0}=\min \mathcal{J}_{t}(\phi)$ and $n_{y}=\operatorname{card} \mathcal{J}_{t}(y)$, we have

(a) for all $j \in \mathcal{J}_{t}(\phi)$ such that $j<j_{0}+n_{y}$, we have $\rho_{y}(j)=\rho_{y}(j-1)+1$, and: if $j=j_{0}$ and $t>0$,

- $\left(z\left(t, j_{0}-1\right), y^{\mathrm{r}}\left(t, j_{0}-1\right)\right) \in \hat{C} \cup \hat{D}$

- $z\left(t, j_{0}\right) \in G_{e}^{0}\left(z\left(t, j_{0}-1\right), y^{\mathrm{r}}\left(t, j_{0}-1\right)\right)$

else

- $\left(z(t, j-1), y^{\mathrm{r}}(t, j-1)\right) \in \operatorname{cl}(\hat{C}) \cup \hat{D}$

- $z(t, j) \in G_{e}\left(z(t, j-1), y^{\mathrm{r}}(t, j-1)\right)$

with

$$
\begin{aligned}
G_{e}^{0}(z, y) & = \begin{cases}z & \text { if }(z, y) \in \hat{C} \backslash \hat{D} \\
G(z, y) & \text { if }(z, y) \in \hat{D} \backslash \hat{C} \\
\{z, G(z, y)\} & \text { if }(z, y) \in \hat{D} \cap \hat{C}\end{cases} \\
G_{e}(z, y) & = \begin{cases}z & \text { if }(z, y) \in \operatorname{cl}(\hat{C}) \backslash \hat{D} \\
G(z, y) & \text { if }(z, y) \in \hat{D} \backslash \operatorname{cl}(\hat{C}) \\
\{z, G(z, y)\} & \text { if }(z, y) \in \hat{D} \cap \operatorname{cl}(\hat{C})\end{cases}
\end{aligned}
$$

(b) for all $j \in \mathcal{J}_{t}(\phi)$ such that $j \geq j_{0}+n_{y}$, we have $\rho_{y}(j)=\rho_{y}(j-1)$ and

- $\left(z(t, j-1), y^{\mathrm{r}}(t, j-1)\right) \in \hat{D}$

- $z(t, j) \in G\left(z(t, j-1), y^{\mathrm{r}}(t, j-1)\right)$ 
5. for all $(t, j) \in \operatorname{dom} \phi, \hat{x}(t, j)=H\left(z(t, j), y^{\mathrm{r}}(t, j)\right)$.

The solution $\phi$ is said to be maximal if there does not exist any other solution $\tilde{\phi}$ such that

$$
\operatorname{dom} \phi \subset \operatorname{dom} \tilde{\phi} \quad, \quad \tilde{\phi}(t, j)=\phi(t, j) \quad \forall(t, j) \in \operatorname{dom} \phi .
$$

$n_{y}$ stands for the number of jumps of $y$ at time $t$. If $n_{y}=0$, jumps of $\phi$ may happen only according to condition $4 \mathrm{~b}$ ) along $G$ if $\phi$ is in $\hat{D}$. Otherwise, if $n_{y}>1$, the jumps of $y$ are recorded consecutively and $\phi$ jumps according to condition $4 \mathrm{a})$ :

- at the first jump happening at $t>0, \phi$ must be in $\hat{C} \cup \hat{D}$ and $z$ is reset either trivially (via the identity) or to a point in $G(z, y)$ according to $G_{e}^{0}$.

- for the remaining jumps of $y$ at $t>0$, or if $t=0$, those conditions are relaxed with $G_{e}$, replacing $\hat{C}$ by $\operatorname{cl}(\hat{C})$.

The difference between $G_{e}^{0}$ and $G_{e}$ in Condition 4a) is that $z$ is forced to jump according to $G$ if $\phi$ is in $\hat{D} \backslash \hat{C}$ instead of $\hat{D} \backslash \operatorname{cl}(\hat{C})$. This stricter condition at the first jump of $y$ after an interval of flow is to avoid the situation where $\phi$ would leave $\hat{C}$ after flow and then be allowed to flow again from the same point after the jump of $y$; namely it prevents flows through a hole of $\hat{C}$. This condition is already enforced when the input does not jump $\left(n_{y}=0\right)$ by conditions 3$)$ and $\left.4 \mathrm{~b}\right)$. In other words, if $\phi$ leaves $\hat{C}$ after an interval of flow, it either jumps according to $G$ if it is in $\hat{D}$ or dies. Hence the condition that $\phi$ should be in $\hat{C} \cup \hat{D}$ instead of $\operatorname{cl}(\hat{C}) \cup \hat{D}$ at the first jump of $y$. On the other hand, for the remaining jumps of $y$ or at $t=0$, there is no reason to force $x$ to jump with $G$ on $\operatorname{cl}(\hat{C}) \backslash \hat{C}$ since $y$ could possibly flow into $\hat{C}$. That is why $G_{e}^{0}$ is relaxed into $G_{e}$. This distinction disappears if $\hat{C}$ is closed. Note that more generally, the solution stops if $\phi$ leaves $\operatorname{cl}(\hat{C}) \cup \hat{D}$.

\section{Appendix B. Other properties of $\mathcal{R}_{c}$}

We first make more precise the definition of $j$-reparametrization.

Definition Appendix B.1. Given two hybrid arcs $x$ and $x^{\mathrm{r}}, x^{\mathrm{r}}$ is a (resp. full) $j$-reparametrization with consecutive jumps of $x$ with reparametrization map $\rho$ if

- it is a (resp. full) $j$-reparametrization of $x$ with reparametrization map $\rho$

- for any $t \in \mathcal{T}\left(x^{\mathrm{r}}\right)$, there exists an integer $n_{x}$ such that denoting $j_{0}=\min \mathcal{J}^{t}\left(x^{\mathrm{r}}\right)$, for any $j \in \mathcal{J}^{t}\left(x^{\mathrm{r}}\right)$,

$$
\rho(j)= \begin{cases}\rho(j-1)+1 & \text { if } j<j_{0}+n_{x} \\ \rho(j-1) & \text { if } j \geq j_{0}+n_{x} .\end{cases}
$$

In other words, the jumps of $x$ are recorded consecutively in $x^{\mathrm{r}}$. This is ensured by $\mathcal{R}_{c}$, so that $\left(x_{a}^{\mathrm{r}}, x_{b}^{\mathrm{r}}\right):=$ $\mathcal{R}_{c}\left(x_{a}, x_{b}\right)$ are actually $j$-reparametrizations with consecutive jumps of $x_{a}$ and $x_{b}$ respectively.

Lemma Appendix B.2. Consider complete hybrid arcs $x_{a}, x_{b}, x_{a, 0}$ and $x_{b, 0}$ such that

$$
\operatorname{dom}_{t} x_{a}=\operatorname{dom}_{t} x_{b}=\operatorname{dom}_{t} x_{a, 0}=\operatorname{dom}_{t} x_{b, 0} .
$$

If $x_{a}, x_{b}$ are full $j$-reparametrizations with consecutive jumps of $x_{a, 0}$ and $x_{b, 0}$ respectively, then, $\mathcal{R}_{c}\left(x_{a}, x_{b}\right)$ is a full $j$-reparametrization of $\mathcal{R}_{c}\left(x_{a, 0}, x_{b, 0}\right)$. 
Proof We denote $T:=T\left(x_{a}\right)=T\left(x_{b}\right)=T\left(x_{a, 0}, x_{b, 0}\right)$. There exist $\rho_{a, 0}, \rho_{b, 0}$ verifying (8) such that

$$
x_{1}(t, j)=x_{a, 0}\left(t, \rho_{a, 0}(j)\right) \quad \forall(t, j) \in \operatorname{dom} x_{1} \quad, \quad x_{b}(t, j)=x_{b, 0}\left(t, \rho_{b, 0}(j)\right) \quad \forall(t, j) \in \operatorname{dom} x_{b}
$$

Denote $\phi^{\mathrm{r}}=\left(x_{a}^{r}, x_{b}^{r}\right)=\mathcal{R}_{c}\left(x_{a}, x_{b}\right)$ and $\phi_{0}^{\mathrm{r}}=\left(x_{a, 0}^{r}, x_{b, 0}^{r}\right)=\mathcal{R}_{c}\left(x_{a, 0}, x_{b, 0}\right) . x_{1}^{\mathrm{r}}$ and $x_{b}^{\mathrm{r}}$ are $j$-reparametrizations with consecutive jumps of $x_{1}$ and $x_{b}$ respectively, and $x_{a, 0}^{r}$ and $x_{b, 0}^{r}$ are $j$-reparametrizations with consecutive jumps of $x_{a, 0}$ and $x_{b, 0}$ respectively: there exist $\rho_{1}^{\mathrm{r}}, \rho_{b}^{\mathrm{r}}, \rho_{a, 0}^{r}, \rho_{b, 0}^{r}$ all verifying (8) such that

$$
\begin{gathered}
x_{1}^{\mathrm{r}}(t, j)=x_{1}\left(t, \rho_{1}^{\mathrm{r}}(j)\right) \quad, \quad x_{b}^{\mathrm{r}}(t, j)=x_{b}\left(t, \rho_{b}^{\mathrm{r}}(j)\right) \quad \forall(t, j) \in \operatorname{dom} \phi^{\mathrm{r}} \\
x_{a, 0}^{r}(t, j)=x_{a, 0}\left(t, \rho_{a, 0}^{r}(j)\right) \quad, \quad x_{b, 0}^{r}(t, j)=x_{b, 0}\left(t, \rho_{b, 0}^{r}(j)\right) \quad \forall(t, j) \in \operatorname{dom} \phi_{0}^{r}
\end{gathered}
$$

Combining (B.2) and (B.1), it follows that

$$
x_{1}^{\mathrm{r}}(t, j)=x_{a, 0}\left(t, \rho_{a, 0} \circ \rho_{1}^{\mathrm{r}}(j)\right) \quad, \quad x_{b}^{\mathrm{r}}(t, j)=x_{b, 0}\left(t, \rho_{b, 0} \circ \rho_{b}^{\mathrm{r}}(j)\right) \quad \forall(t, j) \in \operatorname{dom} \phi^{\mathrm{r}} .
$$

We have to study two cases:

- Case $1: T \notin \operatorname{dom}_{t} \phi^{\mathrm{r}}$

- Case b : $T \in \operatorname{dom}_{t} \phi^{\mathrm{r}}$ and $\operatorname{card} \mathcal{J}_{T}\left(x_{i}\right)=\operatorname{card} \mathcal{J}_{T}\left(x_{i, 0}\right)=+\infty$ for $i=a, b$.

Now, by using the consecutive jumps properties of $\rho_{i, 0}$ and $\rho_{i}^{\mathrm{r}}$, we get that for all $t \in \mathcal{T}\left(\phi^{\mathrm{r}}\right)$, there exist integers $n_{x_{i}^{\mathrm{r}}}$ and $n_{x_{i, 0}}$, such that denoting $j_{0}=\min \mathcal{J}_{t}\left(\phi^{\mathrm{r}}\right)$, we have for all $j \in \mathcal{J}_{t}\left(\phi^{\mathrm{r}}\right)$,

$$
\rho_{i}^{\mathrm{r}}(j)= \begin{cases}\rho_{i}^{\mathrm{r}}(j-1)+1 & \text { if } j<j_{0}+n_{x_{i}^{\mathrm{r}}} \\ \rho_{i}^{\mathrm{r}}(j-1) & \text { if } j \geq j_{0}+n_{x_{i}^{\mathrm{r}}}\end{cases}
$$

and

$$
\rho_{i, 0} \circ \rho_{i}^{\mathrm{r}}(j)=\left\{\begin{array}{ll}
\rho_{i, 0} \circ \rho_{i}^{\mathrm{r}}(j-1)+1 & \text { if } j<j_{0}+n_{x_{i, 0}} \\
\rho_{i, 0} \circ \rho_{i}^{\mathrm{r}}(j-1) & \text { if } j \geq j_{0}+n_{x_{i, 0}}
\end{array} .\right.
$$

In other words, $x_{i}^{\mathrm{r}}$ is a $j$-reparametrization with consecutive jumps of $x_{i, 0}$. Besides, according to (B.4), $n_{x_{i, 0}}=\operatorname{card} \mathcal{J}_{t}\left(x_{i, 0}\right)$, except maybe at $t=T$ where we could have $n_{x_{i, 0}} \leq$ card $\mathcal{J}_{T}\left(x_{i, 0}\right)$ (if $\phi^{\mathrm{r}}$ stopped before browsing all the jumps of $x_{i, 0}$ ), but we know this is not possible because if $T \in \operatorname{dom}_{t}$, all the arcs jump an infinite number of times.

Similarly, by the consecutive jumps properties of $\rho_{i, 0}^{\mathrm{r}}$, we get that for all $t \in \mathcal{T}\left(\phi_{0}^{\mathrm{r}}\right)$, there exist integers $n_{x_{i, 0}^{\mathrm{r}}}$, such that denoting $j_{0}^{\prime}=\min \mathcal{J}_{t}\left(\phi_{0}^{\mathrm{r}}\right)$, we have for all $j^{\prime} \in \mathcal{J}_{t}\left(\phi_{0}^{\mathrm{r}}\right)$,

$$
\rho_{i, 0}^{\mathrm{r}}(j)= \begin{cases}\rho_{i, 0}^{\mathrm{r}}\left(j^{\prime}-1\right)+1 & \text { if } j^{\prime}<j_{0}^{\prime}+n_{x_{i, 0}^{\mathrm{r}}} \\ \rho_{i, 0}^{\mathrm{r}}\left(j^{\prime}-1\right) & \text { if } j^{\prime} \geq j_{0}^{\prime}+n_{x_{i, 0}^{\mathrm{r}}}\end{cases}
$$

From (B.3), again, $n_{x_{i, 0}^{\mathrm{r}}}=\operatorname{card} \mathcal{J}_{t}\left(x_{i, 0}\right)$. Besides, for any $t \in \mathcal{T}\left(\phi^{\mathrm{r}}\right), t \in \mathcal{T}\left(\phi_{0}^{\mathrm{r}}\right)$ if and only if $\max \left\{n_{x_{a, 0}}, n_{x_{b, 0}}\right\} \neq$ 0 . Indeed, $n_{x_{a, 0}}=n_{x_{b, 0}}=0$ means that no jump of $\phi^{\mathrm{r}}$ at time $t$ correspond to any jump of either $x_{a, 0}$ or $x_{b, 0}$ at time $t$. Because of the consecutiveness of the jumps in the reparametrization of $x_{i}^{\mathrm{r}}$ with respect to $x_{i, 0}$, this is equivalent to the fact that no jump occurs in neither of the $x_{i, 0}$, which is equivalent to the fact that no jumps occurs at time $t$ in $\phi_{0}^{\mathrm{r}}$. In other words, we conclude that for all $t$ in $\mathcal{T}\left(\phi^{\mathrm{r}}\right)$ :

- either $t \notin \mathcal{T}\left(\phi_{0}^{\mathrm{r}}\right)$, and $n_{x_{a, 0}}=n_{x_{b, 0}}=0$

- or $t \in \mathcal{T}\left(\phi_{0}^{\mathrm{r}}\right)$, and $n_{x_{i, 0}}=n_{x_{i, 0}^{\mathrm{r}}}$ for $i=a, b$.

Now let us build recursively a function $\bar{\rho}$ with :

- $\bar{\rho}(0)=0$

- for $j$ in $\left\{1, \cdots, J\left(\phi^{\mathrm{r}}\right)\right\} \cap \mathbb{N}$,

$$
\bar{\rho}(j)= \begin{cases}\bar{\rho}(j-1)+1 & \text { if } \rho_{1,0} \circ \rho_{1}^{\mathrm{r}}(j)=\rho_{1,0} \circ \rho_{1}^{\mathrm{r}}(j-1)+1 \text { or } \rho_{b, 0} \circ \rho_{b}^{\mathrm{r}}(j)=\rho_{b, 0} \circ \rho_{b}^{\mathrm{r}}(j-1)+1 \\ \bar{\rho}(j-1) & \text { otherwise }\end{cases}
$$


$\bar{\rho}$ verifies (8) and according to (B.5), we have for all $t$ in $\operatorname{dom}_{t} \phi^{\mathrm{r}}$ and for all $j \in \mathcal{J}_{t}\left(\phi^{\mathrm{r}}\right)$

$$
\bar{\rho}(j)= \begin{cases}\bar{\rho}(j-1)+1 & \text { if } j<j_{0}+\max \left\{n_{x_{a}, 0}, n_{x_{b, 0}}\right\} \\ \bar{\rho}(j-1) & \text { if } j \geq j_{0}+\max \left\{n_{x_{a, 0}}, n_{x_{b, 0}}\right\} .\end{cases}
$$

We would like to prove that

$$
x_{1}^{\mathrm{r}}(t, j)=x_{a, 0}^{\mathrm{r}}(t, \bar{\rho}(j)) \quad, \quad x_{b}^{\mathrm{r}}(t, j)=x_{b, 0}^{\mathrm{r}}(t, \bar{\rho}(j)) \quad \forall(t, j) \in \operatorname{dom} \phi^{\mathrm{r}} .
$$

Let us prove by induction for $j$ in $\operatorname{dom}_{j}\left(\phi^{\mathrm{r}}\right)$ such that $t_{j} \neq T$ that

$$
\mathcal{P}(j): \mathcal{I}_{j}\left(\phi^{\mathrm{r}}\right) \subseteq \mathcal{I}_{\bar{\rho}(j)}\left(\phi_{0}^{\mathrm{r}}\right), \quad \rho_{i, 0} \circ \rho_{1}^{\mathrm{r}}(j)=\rho_{i, 0}^{\mathrm{r}} \circ \bar{\rho}(j), i=a, b
$$

Indeed, from (B.3) and (B.4), $\mathcal{P}(j)$ will directly imply that

$$
\forall t \in \mathcal{I}_{j}\left(\phi^{\mathrm{r}}\right), \quad x_{1}^{\mathrm{r}}(t, j)=x_{a, 0}^{\mathrm{r}}(t, \bar{\rho}(j)), \quad x_{b}^{\mathrm{r}}(t, j)=x_{b, 0}^{\mathrm{r}}(t, \bar{\rho}(j))
$$

which will give (B.8) for $t<T$. If $t_{0}=T$, there is nothing to check. Otherwise, we are going to browse $\operatorname{dom}_{t} \phi^{\mathrm{r}}$ up to $T$, treating together all the jumps occuring at a common time. Start at $t_{0}$ with $j=0: \mathcal{P}(0)$ holds because for $i \in\{a, b\}, \bar{\rho}(0)=\rho_{i, 0} \circ \rho_{i}^{\mathrm{r}}(0)=\rho_{i, 0}^{\mathrm{r}} \circ \bar{\rho}(0)=0$ and $\mathcal{I}_{0}\left(\phi^{\mathrm{r}}\right) \subseteq \mathcal{I}_{0}\left(x_{a, 0}\right) \cap \mathcal{I}_{0}\left(x_{b, 0}\right)=\mathcal{I}_{0}\left(\phi_{0}^{\mathrm{r}}\right)$. Consider the jumps occurring in $\phi^{\mathrm{r}}$ at $t_{0}$ starting from $j_{0}=1$ :

- either $t_{0} \notin \mathcal{T}\left(\phi_{0}^{\mathrm{r}}\right)$, then $n_{x_{a, 0}}=n_{x_{b, 0}}=0$, and recursively, for all $j \in \mathcal{J}_{t_{0}}\left(\phi^{\mathrm{r}}\right), \rho_{i, 0} \circ \rho_{i}^{\mathrm{r}}(j)=\rho_{i, 0} \circ \rho_{i}^{\mathrm{r}}\left(j_{0}-1\right)=$ 0. Therefore also, $\bar{\rho}(j)=\bar{\rho}\left(j_{0}-1\right)=0$ and thus, $\rho_{i, 0}^{\mathrm{r}} \circ \bar{\rho}(j)=\rho_{i, 0}^{\mathrm{r}} \circ \bar{\rho}\left(j_{0}-1\right)=0$.

- either $t_{0} \in \mathcal{T}\left(\phi_{0}^{\mathrm{r}}\right), j_{0}^{\prime}=1=\bar{\rho}\left(j_{0}\right), n_{x_{i, 0}}=n_{x_{i, 0}^{\mathrm{r}}}$ : without loss of generality assume $n_{x_{a, 0}} \leq n_{x_{b, 0}}$, then

- for all $j<j_{0}+n_{x_{a, 0}}$, we have $\rho_{i, 0} \circ \rho_{i}^{\mathrm{r}}(j)=\rho_{i, 0} \circ \rho_{i}^{\mathrm{r}}(j-1)+1, \bar{\rho}(j)=\bar{\rho}(j-1)+1$, and thus also $\rho_{i, 0}^{\mathrm{r}} \circ \bar{\rho}(j)=\rho_{i, 0}^{\mathrm{r}} \circ \bar{\rho}(j-1)+1$ with $j^{\prime}=\bar{\rho}(j)$.

- for all $j_{0}+n_{x_{a, 0}} \leq j<j_{0}+n_{x_{b, 0}}$, we have $\rho_{a, 0} \circ \rho_{1}^{\mathrm{r}}(j)=\rho_{a, 0} \circ \rho_{1}^{\mathrm{r}}(j-1), \rho_{b, 0} \circ \rho_{b}^{\mathrm{r}}(j)=\rho_{b, 0} \circ \rho_{b}^{\mathrm{r}}(j-1)+1$, therefore $\bar{\rho}(j)=\bar{\rho}(j-1)+1$, and with $j^{\prime}=\bar{\rho}(j)$, we get $\rho_{a, 0}^{\mathrm{r}} \circ \bar{\rho}(j)=\rho_{a, 0}^{\mathrm{r}} \circ \bar{\rho}(j-1)$ and $\rho_{b, 0}^{\mathrm{r}} \circ \bar{\rho}(j)=$ $\rho_{b, 0}^{\mathrm{r}} \circ \bar{\rho}(j-1)+1$.

- for all $j \geq j_{0}+n_{x_{b, 0}}$, we have $\rho_{i, 0} \circ \rho_{i}^{\mathrm{r}}(j)=\rho_{i, 0} \circ \rho_{i}^{\mathrm{r}}(j-1), \bar{\rho}(j)=\bar{\rho}(j-1)$, and thus also $\rho_{i, 0}^{\mathrm{r}} \circ \bar{\rho}(j)=$ $\rho_{i, 0}^{\mathrm{r}} \circ \bar{\rho}(j-1)$.

Therefore, in all cases, $\rho_{i, 0} \circ \rho_{i}^{\mathrm{r}}(j)=\rho_{i, 0}^{\mathrm{r}} \circ \bar{\rho}(j)$ for all $j \in \mathcal{J}_{t_{0}}\left(\phi^{\mathrm{r}}\right)$. Besides, since $t_{0} \neq T$, there is a finite number of jumps at that time. Up to the last jump, $\mathcal{I}_{j}\left(\phi^{\mathrm{r}}\right)=\left\{t_{0}\right\} \subseteq \mathcal{I}_{\bar{\rho}(j)}\left(\phi_{0}^{\mathrm{r}}\right)$. As for the last jump, $\mathcal{I}_{j}\left(\phi^{\mathrm{r}}\right)$ is of non-empty interval (still because $t_{0} \neq T$ ), and by definition of the reparametrization,

$$
\mathcal{I}_{j}\left(\phi^{\mathrm{r}}\right) \subseteq \mathcal{I}_{\rho_{a, 0} \circ \rho_{1}^{\mathrm{r}}(j)}\left(x_{a, 0}\right) \cap \mathcal{I}_{\rho_{b, 0} \circ \rho_{b}^{\mathrm{r}}(j)}\left(x_{b, 0}\right)=\mathcal{I}_{\rho_{a, 0}^{\mathrm{r}} \circ \bar{\rho}}\left(x_{a, 0}\right) \cap \mathcal{I}_{\rho_{b, 0}^{\mathrm{r}} \circ \bar{\rho}}\left(x_{b, 0}\right)=\mathcal{I}_{\bar{\rho}(j)}\left(\phi_{0}^{\mathrm{r}}\right) .
$$

Therefore, $\mathcal{P}(j)$ holds for all $j \in \mathcal{J}_{t_{0}}\left(\phi^{\mathrm{r}}\right)$. Moving along the successive jump times $t \in \mathcal{T}\left(\phi^{\mathrm{r}}\right)$, we manage to browse $\operatorname{dom}_{t}\left(\phi^{\mathrm{r}}\right)$ up to $T$. Therefore, (B.8) holds for all $(t, j)$ with $t<T$.

If $T \notin \operatorname{dom}_{t}\left(\phi^{\mathrm{r}}\right)$ (Case 1), (B.8) is proved. If $T \in \operatorname{dom}_{t}\left(\phi^{\mathrm{r}}\right)\left(\right.$ Case b) and at $t=T, n_{x_{1}}=n_{x_{b}}=n_{x_{1}^{\mathrm{r}}}=$ $n_{x_{b}^{\mathrm{r}}}=$ card $J^{T}\left(\phi^{\mathrm{r}}\right)=+\infty$, and the result follows in the same way.

We thus deduce that (B.8) holds i.e.

$$
\phi^{\mathrm{r}}(t, j)=\phi_{0}^{\mathrm{r}}(t, \bar{\rho}(j)) \quad \forall(t, j) \in \operatorname{dom} \phi^{\mathrm{r}}
$$

and $\phi^{\mathrm{r}}$ is a $j$-reparametrization of $\phi_{0}^{\mathrm{r}}$. Remains to prove that this reparametrization is full. Since $\operatorname{dom}_{t} \phi^{\mathrm{r}}=$ $\operatorname{dom}_{t} \phi_{0}^{\mathrm{r}}$, all the values of $\phi_{0}^{\mathrm{r}}$ appear in $\phi^{\mathrm{r}}$ except maybe those at the boundary of the time domain, i.e those at time $T$, if $T \in \operatorname{dom}_{t} \phi_{0}^{\mathrm{r}}=\operatorname{dom}_{t} \phi^{\mathrm{r}}$. But any jump occurring at time $T$ in $\phi_{0}^{\mathrm{r}}$ is present either in $x_{a, 0}$ or $x_{b, 0}$, and therefore in either $x_{a}$ or $x_{b}$ by full-reparametrization, and therefore in $\phi^{\mathrm{r}}$ by definition of $\mathcal{R}_{c}$. 


\section{Appendix C. Properties of $\mathcal{R}_{c}\left(\mathcal{H}_{a}, \mathcal{H}_{b}\right)$}

Similarly to the definition of $\mathcal{H}^{\mathrm{r}}:=\mathcal{R}_{c}(\mathcal{H}, \mathcal{H})$ in (18), we define $\mathcal{R}_{c}\left(\mathcal{H}_{a}, \mathcal{H}_{b}\right)$ such that $\mathcal{R}_{c}\left(x_{a}, x_{b}\right)$ is solution to $\mathcal{R}_{c}\left(\mathcal{H}_{a}, \mathcal{H}_{b}\right)$ when $x_{a}$ (resp. $\left.x_{b}\right)$ is solution to $\mathcal{H}_{a}$ (resp. $\left.\mathcal{H}_{b}\right)$.

Definition Appendix C.1. Given two hybrid systems $\mathcal{H}_{a}=\left(C_{a}, f_{a}, D_{a}, g_{a}\right)$ and $\mathcal{H}_{b}=\left(C_{b}, f_{b}, D_{b}, g_{b}\right)$, we define $\mathcal{R}_{c}\left(\mathcal{H}_{a}, \mathcal{H}_{b}\right)$ as the hybrid system

$$
\mathcal{H}^{\mathrm{r}}\left\{\begin{array}{cc}
\left(\begin{array}{c}
\dot{x}_{a} \\
\dot{x}_{b}
\end{array}\right) \in f^{\mathrm{r}}\left(x_{a}, x_{b}\right) & \left(x_{a}, x_{b}\right) \in C^{\mathrm{r}} \\
\left(\begin{array}{c}
x_{a}^{+} \\
x_{b}^{+}
\end{array}\right) \in g^{\mathrm{r}}\left(x_{a}, x_{b}\right) & \left(x_{a}, x_{b}\right) \in D^{\mathrm{r}}
\end{array}\right.
$$

with

$$
C^{\mathrm{r}}=C_{a} \times C_{b} \quad, \quad D^{\mathrm{r}}=\left(D_{a} \times\left(\operatorname{cl}\left(C_{b}\right) \cup D_{b}\right)\right) \cup\left(\left(\operatorname{cl}\left(C_{a}\right) \cup D_{a}\right) \times D_{b}\right)
$$

and

$$
\begin{aligned}
& f^{\mathrm{r}}\left(x_{a}, x_{b}\right)=\left(\begin{array}{c}
f_{a}\left(x_{a}\right) \\
f_{b}\left(x_{b}\right)
\end{array}\right) \quad \forall\left(x_{a}, x_{b}\right) \in C^{\mathrm{r}} \\
& g^{\mathrm{r}}\left(x_{a}, x_{b}\right)=\left(\begin{array}{c}
g \\
\underline{g}_{a}\left(x_{a}\right) \\
\underline{\operatorname{Id}}_{b}\left(x_{b}\right)
\end{array}\right) \cup\left(\begin{array}{c}
\underline{\operatorname{Id}}_{a}\left(x_{a}\right) \\
\underline{g}_{b}\left(x_{b}\right)
\end{array}\right) \cup\left(\begin{array}{c}
\underline{g}_{a}\left(x_{a}\right) \\
\underline{g}_{b}\left(x_{b}\right)
\end{array}\right) \quad \forall\left(x_{a}, x_{b}\right) \in D^{\mathrm{r}}
\end{aligned}
$$

where we have denoted for $i$ in $\{1, b\}$

$$
\underline{g}_{i}\left(x_{i}\right)=\left\{\begin{array}{ll}
g_{i}\left(x_{i}\right) & \text { if } x_{i} \in D_{i} \\
\emptyset & \text { otherwise }
\end{array} \quad, \quad \underline{\operatorname{Id}}_{i}\left(x_{i}\right)=\left\{\begin{array}{ll}
x_{i} & \text { if } x_{i} \in \operatorname{cl}\left(C_{i}\right) \\
\emptyset & \text { otherwise }
\end{array} .\right.\right.
$$

In the definition (C.4) of the jump map of $\mathcal{R}_{c}\left(\mathcal{H}_{a}, \mathcal{H}_{b}\right)$, we allow $x_{a}$ (resp. $x_{b}$ ) to be reset trivially even when both $x_{a}$ and $x_{b}$ are in their jump sets $D_{a}$ and $D_{b}$, instead of making them jump simultaneously with their jump map $g_{i}$. This is necessary because $x_{i}$ could be flowing $D_{i}$ int $C_{i}$ while the other jump (unless no flow is possible there).

However, this introduces solutions $\left(x_{a}^{\mathrm{r}}, x_{b}^{\mathrm{r}}\right)$ to $\mathcal{H}^{\mathrm{r}}$ that cannot be written as $\mathcal{R}_{c}\left(x_{a}, x_{b}\right)$ for any $x_{a}$ and $x_{b}$ solutions to $\mathcal{H}_{a}$ and $\mathcal{H}_{b}$. Indeed, when $x_{a}$ and $x_{b}$ jump simultaneously, $\mathcal{R}_{c}\left(x_{a}, x_{b}\right)$ jumps only once, whereas it would be allowed by the definition of $\mathcal{H}^{\mathrm{r}}$ that $x_{a}^{\mathrm{r}}$ and $x_{b}^{\mathrm{r}}$ jump according to $g_{a}$ and $g_{b}$ one after the other. However, since the two hybrid systems are decoupled, the only addition is a trivial jump in the state component that does not jump.

Therefore, before relating the solutions to $\mathcal{H}_{a}$ and $\mathcal{H}_{b}$ and $\mathcal{R}_{c}\left(\mathcal{H}_{a}, \mathcal{H}_{b}\right)$, we need the following definition.

Definition Appendix C.2. Consider a solution $\phi^{\mathrm{r}}=\left(x_{a}^{\mathrm{r}}, x_{b}^{\mathrm{r}}\right)$ to $\mathcal{R}_{c}\left(\mathcal{H}_{a}, \mathcal{H}_{b}\right)$. At a time $t$ in $\mathcal{T}\left(\phi^{\mathrm{r}}\right)$ and at a jump $j \in \mathcal{J}^{t}\left(\phi^{\mathrm{r}}\right)$, we say that $x_{i}^{\mathrm{r}}$ satisfies its jump condition if

$$
x_{i}^{\mathrm{r}}(t, j-1) \in D_{i} \text { and } x_{i}^{\mathrm{r}}(t, j) \in g_{i}\left(x_{a}^{\mathrm{r}}(t, j-1)\right) .
$$

We introduce the following conditions:

Condition 1. For any $t$ in $\mathcal{T}\left(\phi^{\mathrm{r}}\right)$, there exist integers $n_{x_{i}}$ such that, for all $j \in \mathcal{J}^{t}\left(\phi^{\mathrm{r}}\right)$, denoting $j_{0}=$ $\min \mathcal{J}^{t}\left(\phi^{\mathrm{r}}\right)$,

- $x_{i}^{\mathrm{r}}$ satisfies its jump condition if $j<j_{0}+n_{x_{i}}$.

- $x_{i}^{\mathrm{r}}$ does not satisfy its jump condition if $j \geq j_{0}+n_{x_{i}}$. 
Condition 2. for any $t$ in $\mathcal{T}\left(\phi^{\mathrm{r}}\right) \cap \operatorname{int} \operatorname{dom}_{t}\left(\phi^{\mathrm{r}}\right)$, if $x_{i}^{\mathrm{r}}$ does not verify its jump condition for any $j \in \mathcal{J}^{t}\left(\phi^{\mathrm{r}}\right)$, then $x_{i}^{\mathrm{r}}(t, j) \in C_{i}$ for all $j \in \mathcal{J}^{t}\left(\phi^{\mathrm{r}}\right)$.

Condition 1 is a consecutive jump condition that says that at each jump time of $\phi^{\mathrm{r}}, x_{a}^{\mathrm{r}}$ and $x_{b}^{\mathrm{r}}$ first jump simultaneously according to their jump condition and then stay constant until the other has completed all its jumps. In other words, they jump simultaneously and consecutively, but not alternatively.

Condition 2 requires that at each time $t$ where $x_{i}^{\mathrm{r}}$ never jumps according to its jump condition, $x_{i}^{\mathrm{r}}$ is in $C_{i}$. This condition automatically holds for $x_{i}^{\mathrm{r}}$ when $C_{i}$ is closed.

The following technical lemma relates the solutions to $\mathcal{H}_{a}$ and $\mathcal{H}_{b}$ and $\mathcal{R}_{c}\left(\mathcal{H}_{a}, \mathcal{H}_{b}\right)$.

Lemma Appendix C.3. Consider two hybrid systems $\mathcal{H}_{a}=\left(C_{a}, f_{a}, D_{a}, g_{a}\right)$ and $\mathcal{H}_{b}=\left(C_{b}, f_{b}, D_{b}, g_{b}\right)$.

- For any solutions $x_{a}$ to $\mathcal{H}_{a}$ and $x_{b}$ to $\mathcal{H}_{b}$, the hybrid $\operatorname{arc} \phi^{\mathrm{r}}:=\left(x_{a}^{\mathrm{r}}, x_{b}^{\mathrm{r}}\right):=\mathcal{R}_{c}\left(x_{a}, x_{b}\right)$ verifies

- $\phi^{\mathrm{r}}$ is solution to the hybrid system $\mathcal{R}_{c}\left(\mathcal{H}_{a}, \mathcal{H}_{b}\right)$ on its domain $\operatorname{dom} \phi^{\mathrm{r}}=\mathcal{R}_{c}\left(\operatorname{dom} x_{a}, \operatorname{dom} x_{b}\right)$, and $\phi^{\mathrm{r}}$ is maximal for $\mathcal{R}_{c}\left(\mathcal{H}_{a}, \mathcal{H}_{b}\right)$ if $x_{a}$ is maximal for $\mathcal{H}_{a}$ and $x_{b}$ is maximal for $\mathcal{H}_{b}$.

- Conditions 1 and 2 hold for both $x_{a}^{\mathrm{r}}$ and $x_{b}^{\mathrm{r}}$.

- Conversely, for any solution $\phi^{\mathrm{r}}=\left(x_{a}^{\mathrm{r}}, x_{b}^{\mathrm{r}}\right)$ to $\mathcal{R}_{c}\left(\mathcal{H}_{a}, \mathcal{H}_{b}\right)$, if $x_{i}^{\mathrm{r}}$ satisfies Condition 2, there exists $x_{i}$ solution to $\mathcal{H}_{i}$ such that

- $\operatorname{dom}_{t}\left(x_{i}\right)=\operatorname{dom}_{t}\left(\phi^{\mathrm{r}}\right)$ and

$$
J\left(x_{i}\right)=\operatorname{card}\left\{j \in \mathbb{N}_{>0} \cap \operatorname{dom}_{j} \phi^{\mathrm{r}}: x_{i}^{\mathrm{r}}\left(t_{j}, j-1\right) \in D_{i}, x_{i}^{\mathrm{r}}\left(t_{j}, j\right) \in g_{i}\left(x_{i}^{\mathrm{r}}\left(t_{j}, j-1\right)\right)\right\}
$$

- $x_{i}^{\mathrm{r}}$ is a full $j$-reparametrization of $x_{i}$.

Proof Take solutions $x_{a}$ to $\mathcal{H}_{a}$ and $x_{b}$ to $\mathcal{H}_{b}$. The fact that $\mathcal{R}_{c}\left(x_{a}, x_{b}\right)$ is solution to $\mathcal{R}_{c}\left(\mathcal{H}_{a}, \mathcal{H}_{b}\right)$ follows from the following points:

- flow intervals of $\mathcal{R}_{c}\left(x_{a}, x_{b}\right)$ are included in flow intervals of $x_{a}$ and $x_{b}$.

- $\mathcal{R}_{c}\left(x_{a}, x_{b}\right)$ stops whenever either $x_{a}$ or $x_{b}$ stops, so in particular if $x_{a}$ (resp $\left.x_{b}\right)$ jumps outside of $\operatorname{cl}\left(C_{a}\right) \cup D_{a}$ $\left(\right.$ resp cl $\left.\left(C_{b}\right) \cup D_{b}\right)$. Therefore, $\mathcal{R}_{c}\left(x_{a}, x_{b}\right)$ remains in $\operatorname{cl}\left(C^{\mathrm{r}}\right) \cup D^{\mathrm{r}}=\operatorname{cl}\left(C_{a}\right) \cup D_{a} \cup \operatorname{cl}\left(C_{b}\right) \cup D_{b}$ until it stops.

- $\mathcal{R}_{c}\left(x_{a}, x_{b}\right)$ jumps only when either $x_{a}$ or $x_{b}$ jumps, so when at least one $x_{i}$ is in $D_{i}$ and jumps according to $g_{i}$. With the previous point, we deduce that the jumps of $\mathcal{R}_{c}\left(x_{a}, x_{b}\right)$ happen in $D^{\mathrm{r}}$. Besides, $x_{i}$ necessarily jumps according to $g_{i}$ when in $D_{i} \backslash \operatorname{cl}\left(C_{i}\right)$ so that trivial jumps happen only in $\operatorname{cl}\left(C_{i}\right)$. Therefore, $\mathcal{R}_{c}\left(x_{a}, x_{b}\right)$ jumps according to $g^{\mathrm{r}}$.

Also, $\mathcal{R}_{c}\left(x_{a}, x_{b}\right)$ browses the full domain of either $x_{a}$ or $x_{b}$. If $x_{a}$ is maximal for $\mathcal{H}_{a}$ and $x_{b}$ is maximal for $\mathcal{H}_{b}$, then $\mathcal{R}_{c}\left(x_{a}, x_{b}\right)$ cannot be extended either and it is maximal for $\mathcal{R}_{c}\left(\mathcal{H}_{a}, \mathcal{H}_{b}\right)$. Also by construction, at each time $t$ in $\mathcal{T}\left(x_{a}\right) \cap \mathcal{T}\left(x_{b}\right), x_{a}^{\mathrm{r}}$ and $x_{b}^{\mathrm{r}}$ jump according to $g_{a}$ and $g_{b}$ respectively, until all possible jumps of $x_{a}$ or $x_{b}$ have been processed, and afterwards stay constant if the solution carries on until all remaining jumps have been processed. Therefore, $\mathcal{R}_{c}\left(x_{a}, x_{b}\right)$ satisfies Condition 1. As for Condition 2, it follows from the fact that if the jump condition of $x_{i}$ is not verified at any jump of $x_{i}^{\mathrm{r}}$ at a given time $t$ in the interior of the time domain, then those jumps happen in the interior of a flow interval of $x_{i}$, which is therefore in $C_{i}$ by definition of solutions.

Conversely, take $\phi^{\mathrm{r}}=\left(x_{a}^{\mathrm{r}}, x_{b}^{\mathrm{r}}\right)$ solution to $\mathcal{R}_{c}\left(\mathcal{H}_{a}, \mathcal{H}_{b}\right)$. We build two hybrid arcs $x_{a}, x_{b}$ in the following way :

1. start with $\mathcal{D}_{a}=\mathcal{D}_{b}=\mathcal{I}_{0}\left(\phi^{\mathrm{r}}\right) \times\{0\}, x_{a} \equiv x_{1 \mid \mathcal{D}_{a}}^{\mathrm{r}}$ and $x_{b} \equiv x_{b \mid \mathcal{D}_{b}}^{\mathrm{r}}, j_{a}=0, j_{b}=0, \rho_{1}(0)=0$ and $\rho_{b}(0)=0$.

2. for $j$ from 1 to $J\left(\phi^{\mathrm{r}}\right)$ do (denoting $t_{j}=t_{j}\left(\phi^{\mathrm{r}}\right)$ to simplify the notations) : 
- if $x_{a}^{\mathrm{r}}\left(t_{j}, j-1\right) \in D_{a}$ and $x_{a}^{\mathrm{r}}\left(t_{j}, j\right) \in g_{a}\left(x_{a}^{\mathrm{r}}\left(t_{j}, j-1\right)\right), j_{a} \leftarrow j_{a}+1$

- if $x_{b}^{\mathrm{r}}\left(t_{j}, j-1\right) \in D_{b}$ and $x_{b}^{\mathrm{r}}\left(t_{j}, j\right) \in g_{b}\left(x_{b}^{\mathrm{r}}\left(t_{j}, j-1\right)\right), j_{b} \leftarrow j_{b}+1$

- $\mathcal{D}_{a} \leftarrow \mathcal{D}_{a} \cup\left(\mathcal{I}_{j}\left(\phi^{\mathrm{r}}\right) \times\left\{j_{a}\right\}\right)$

- $\mathcal{D}_{b} \leftarrow \mathcal{D}_{b} \cup\left(\mathcal{I}_{j}\left(\phi^{\mathrm{r}}\right) \times\left\{j_{b}\right\}\right)$

- $x_{a}\left(t, j_{a}\right) \leftarrow x_{a}^{\mathrm{r}}(t, j)$ for all $t$ in $\mathcal{I}_{j}\left(\phi^{\mathrm{r}}\right)$

- $x_{b}\left(t, j_{b}\right) \leftarrow x_{b}^{\mathrm{r}}(t, j)$ for all $t$ in $\mathcal{I}_{j}\left(\phi^{\mathrm{r}}\right)$

- $\rho_{1}(j) \leftarrow j_{a}$

- $\rho_{b}(j) \leftarrow j_{b}$

For $i=a, b, x_{i}$ thus built clearly verify the jump conditions of $\mathcal{H}_{i}$. Besides, $x_{i}$ is absolutely continuous during flow, solution to $f_{i}$ almost everywhere, and more importantly $x_{i}$ is in $C_{i}$ in the interior of the flow intervals if Condition 2 holds. In that case, $x_{i}$ is therefore solution to $\mathcal{H}_{i}$ and $\rho_{i}$ is the $j$-reparametrization map from $x_{i}$ to $x_{i}^{\mathrm{r}}$. It is obviously a full $j$-reparametrization since the $\operatorname{arc} x_{i}$ is only defined as long as $\phi^{\mathrm{r}}$ is.

\section{References}

[1] V. Andrieu, G. Besançon, and U. Serres. Observability necessary conditions for the existence of observers. IEEE Conference on Decision and Control, pages 4442-4447, 2013.

[2] G. Xie and L. Wang. Necessary and sufficient conditions for controllability and observability of switched impulsive control systems. IEEE Transactions on Automatic Control, 49(6):960-966, 2004.

[3] E. A. Medina and D. A. Lawrence. Reachability and observability of linear impulsive systems. Automatica, 44:1304-1309, 2008.

[4] A. Tanwani, H. Shim, and D. Liberzon. Comments on "observability of switched linear systems: Characterization and observer design". IEEE Transactions on Automatic Control, 60(12):3396-3400, 2015.

[5] J. Kim, H. Shim, and J. H. Seo. State estimation and tracking control for hybrid systems by gluing the domains. IEEE Transactions on Automatic Control, 64(7):3026-3033, 2019.

[6] F. Forni, A. R. Teel, and L. Zaccarian. Follow the bouncing ball : global results on tracking and state estimation with impacts. IEEE Transactions on Automatic Control, 58(6):1470-1485, 2013.

[7] A. Balluchi, L. Benvenutia, M. D. Di Benedetto, and A. Sangiovanni-Vincentelli. The design of dynamical observers for hybrid systems: Theory and application to an automotive control problem. Automatica, 49(4):915-925, 2013.

[8] C. Lee, Z. Ping, and H. Shim. On-line switching signal estimation of switched linear systems with measurement noise. European Control Conference, 2013.

[9] D. Gómez-Gutiérrez, S. Celikovský, A. Ramírez-Treviño, and B. Castillo-Toledo. On the observer design problem for continuoustime switched linear systems with unknown switchings. Journal of the Franklin Institute, 352(4):1595-1612, 2015.

[10] Z. Ping, C. Lee, and H. Shim. Robust estimation algorithm for both switching signal and state of switched linear systems. International Journal of Control, Automation and Systems, 15(1):95-103, 2017.

[11] R. Vidal, A. Chiuso, S. Soatto, and S. Sastry. Observability of linear hybrid systems. In O. Maler and A. Pnueli, editors, Hybrid Systems: Computation and Control, pages 526-539. Springer Berlin Heidelberg, 2003.

[12] F. Küsters and S. Trenn. Switch observability for switched linear systems. Automatica, 87:121-127, 2017.

[13] R. G. Sanfelice, R. Goebel, and A. R. Teel. Invariance principles for hybrid systems with connections to detectability and asymptotic stability. IEEE Transactions on Automatic Control, 52(12):2282-2297, 2007.

[14] C. Cai and A. R. Teel. Output-to-state stability for hybrid systems. Systems EG Control Letters, 60:62-68, 2011.

[15] P. Bernard and R. G. Sanfelice. An algorithm to generate solutions to hybrid dynamical systems with inputs. 2019 American Control Conference, pages 2996-3001, 2019.

[16] P. Bernard and R. G. Sanfelice. Hybrid dynamical systems with hybrid inputs: definition of solutions and applications to series interconnections. International Journal of Robust and Nonlinear Control, pages 1-25, 2019.

[17] B. Biemond, N. van de Wouw, M.H. Heemels, and H. Nijmeijer. Tracking control for hybrid systems with state-triggered jumps. IEEE Transactions on Automatic Control, 58(4):876-890, 2013.

[18] J.J B. Biemond, R. Postoyan, W.P.M.H. Heemels, and N. Van de Wouw. Incremental stability of hybrid dynamical systems. IEEE Transactions on Automatic Control, 111(108662), 2018.

[19] J.J B. Biemond, R. Postoyan, W.P.M.H. Heemels, and N. Van de Wouw. On the graphical stability of hybrid solutions with non-matching jump times. Automatica, 63(12):4094-4109, 2020.

[20] R. Goebel, R. G. Sanfelice, and A. Teel. Hybrid Dynamical Systems : Modeling, Stability and Robustness. Princeton University Press, 2012.

[21] C. Cai and A. R. Teel. Characterizations of input-to-state stability for hybrid systems. Systems $\&$ Control Letters, 58:47-53, 2009. 
[22] Y. Li and R. G. Sanfelice. Feedback Stabilization of Controlled Dynamical Systems, volume 473 of Lecture Notes in Control and Information Sciences, chapter Incremental Graphical Asymptotic Stability for Hybrid Dynamical Systems, pages 231-262. 2017.

[23] P. Bernard and R. G. Sanfelice. Observers for hybrid dynamical systems with linear maps and known jump times. IEEE Conference on Decision and Control, pages 3140-3145, 2018. 